\title{
Supporting Information for Stable Picodisc Assemblies from Saposin Proteins and Branched Detergents
}

Kathleen W. Kurgan $\uparrow$, Bifan Chen $\uparrow+\S$, Kyle A. Brown $\uparrow+\S$, Paulo F. Cobra \pm , Xinyu Ye $\uparrow$, Ying Ge $† \$$, and Samuel H. Gellman $\dagger^{*}$

$\dagger$ Department of Chemistry, $\ddagger$ Department of Cell and Regenerative Biology, §Human Proteomics Program, and \pm National Magnetic Resonance Facility at Madison, University of Wisconsin-Madison, Madison, Wisconsin 53706, United States

Protein Sequence Design. S2-4

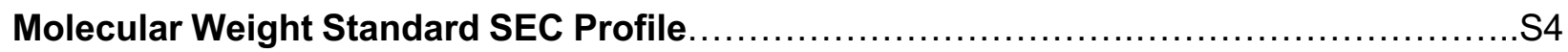

Tryptophan Fluorescence Emission Experimental Details................................ 4 -5

Saposin A+Detergent Tryptophan Fluorescence Emission Data.............................S6

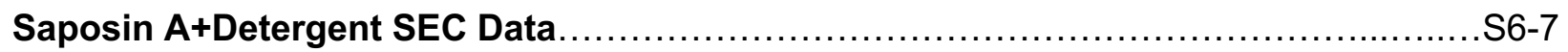

Saposin A+Detergent Native Mass Spectrometry Data ........................................ 8

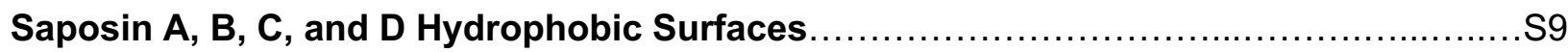

Saposin $\mathrm{C}^{\mathrm{W}}+$ Detergent Tryptophan Fluorescence Emission Data ........................... 10

Saposin $D^{W}+$ Detergent Tryptophan Fluorescence Emission Data........................S10-11

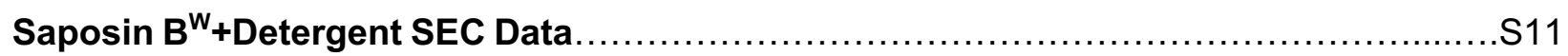

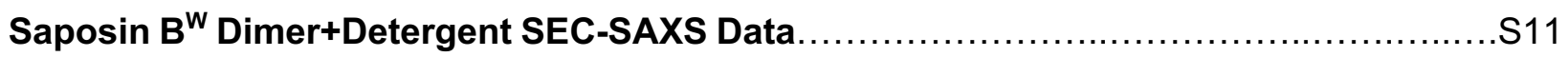

DAMMIF Models Generated From SEC-SAXS Data ......................................... $12-13$

Summary of Saposin $A / B^{W}+$ Detergent Native Mass Spectrometry and SEC-SAXS

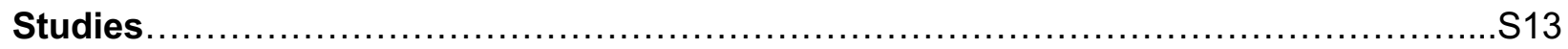

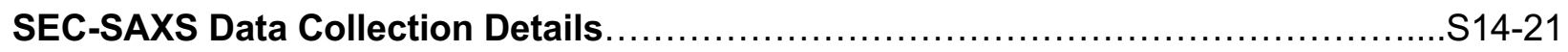

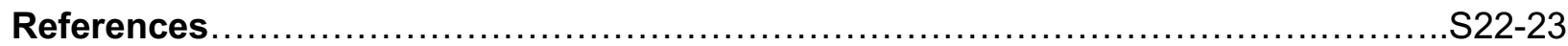


Protein Sequence Design: The DNA sequence encoding saposin A ( $H$. sapiens, Uniprot entry P07602) was codon-optimized by GenScript for expression in E. coli. The DNA sequences encoding saposin B (H. sapiens, Uniprot entry P07602), C (H. sapiens, Uniprot entry P07602), and D (M. musculus, Uniprot entry Q61207) were codon-optimized by GeneUniversal for expression in E. coli. A stop codon was appended to the end of each sequence (highlighted in red), and a tryptophan residue was engineered into the sequences of saposin $B, C$, and $D$. The tryptophan residue was introduced in order to facilitate tryptophan fluorescence emission measurements. The tryptophan residue was introduced at the position within the dimeric structures of saposin $\mathrm{B}, \mathrm{C}$, and D (PBD $1 \mathrm{~N} 69,{ }^{1} 2 \mathrm{QYP},{ }^{2}$ and $\left.5 \mathrm{U} 85^{3}\right)$ that overlaid best with the native tryptophan position in the open structure of saposin $A(P D B 4 D D J 4)$. Figure $S 1$ shows each over the overlay comparisons. The modified sequences are designated saposin $\mathrm{B}^{\mathrm{W}}, \mathrm{C}^{\mathrm{W}}$, and $\mathrm{D}^{\mathrm{w}}$.
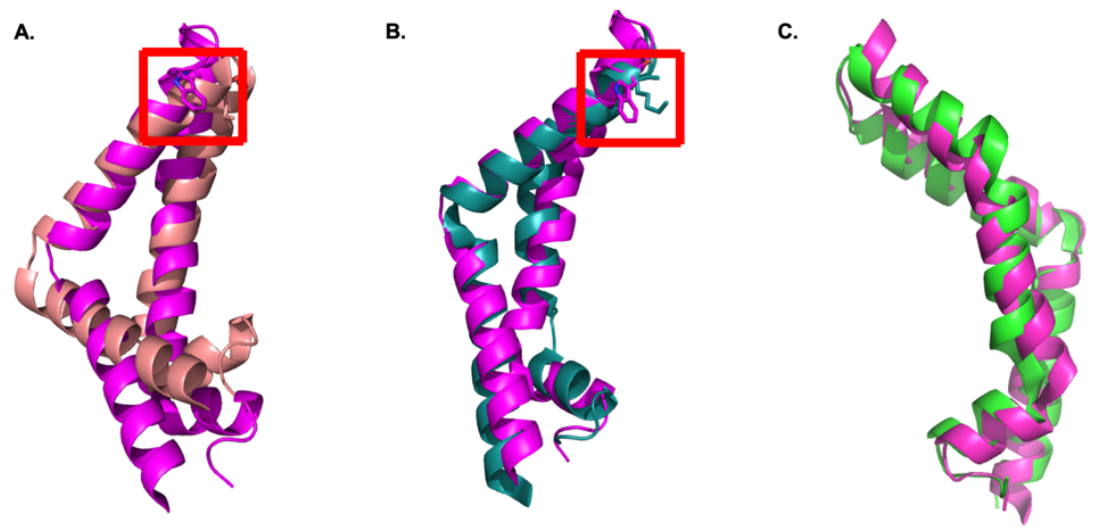

D.

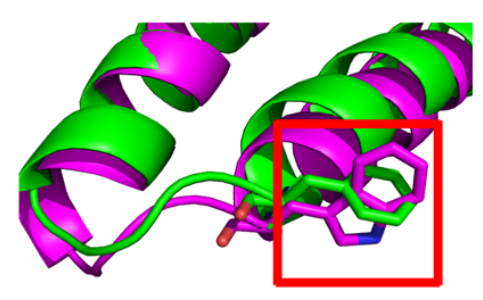

Figure S1. Overlays of the open conformation of saposin A (PDB 4DDJ, magenta) with saposin B (Panel A, salmon, PDB 1N69), C (Panel B, teal, PDB 2QYP), and D (Panels C and D, green, PDB 5U85). The single tryptophan in the saposin A structures and the residues chosen for mutation to tryptophan in the saposin B, C, and D structures are displayed as sticks and boxed in red. 
Below are the sequences of each protein construct described in this work. Each construct consists of a $6 \mathrm{X}-\mathrm{His}$ tag (highlighted in blue) and a thrombin cleavage site (highlighted in yellow). The mutated tryptophan positions are highlighted in pink and enzyme digestion sites in green.

\section{saposin A:}

ATGGGCAGCAGCCATCATCATCATCATCACAGCAGCGGCCTGGTGCCGCGCGGCAGCCA TATGCTCGAGAGCCTGCCGTGCGACATCTGCAAGGATGTGGTTACCGCGGCGGGTGACAT GCTGAAAGATAACGCGACCGAGGAAGAGATTCTGGTGTACCTGGAAAAGACCTGCGACTG GCTGCCGAAACCGAACATGAGCGCGAGCTGCAAGGAGATCGTGGACAGCTATCTGCCGG TTATCCTGGATATCATCAAGGGTGAAATGAGCCGTCCGGGCGAGGTTTGCAGCGCGCTGA ACCTGTGCGAAAGCTAAGGATCCGGCTGCTAACAAAGCCCGA saposin $\mathrm{B}^{\mathrm{w}}$ :

ATGGGCAGCAGCCATCATCATCATCATCACAGCAGCGGCCTGGTGCCGCGCGGCAGCCA TATGGGTGACGTTTGCCAAGATTGTATTCAAATGGTTACCGATATTCAGACCGCCGTTCGT ACCAATTCTACCTTTGTTCAAGCCTTGGTTGAACATGTTAAAGAAGAATGTGATTGGTTGGG TCCTGGTATGGCCGATATTTGCAAAAATTATATTAGCCAGTATAGCGAAATCGCTATTCAGA TGATGATGCATATGCAACCGAAAGAAATTTGTGCTTTGGTTGGTTTTTGTGATTAACTCGAG saposin $\mathrm{C}^{\mathrm{W}}$ :

ATGGGCAGCAGCCATCATCATCATCATCACAGCAGCGGCCTGGTGCCGCGCGGCAGCCA TATGAGCGATGTTTATTGCGAAGTTTGCGAATTTTTGGTTAAAGAAGTTACCAAACTGATCG ATAATAACAAAACCGAAAAGGAAATCTTGGATGCCTTTGATAAAATGTGCAGCTGGTTGCCT AAATCTTTGTCTGAAGAATGCCAGGAAGTTGTTGATACCTATGGCTCTAGCATTTTGAGCAT TTTGTTGGAAGAAGTTTCTCCGGAATTGGTTTGTTCTATGTTGCATTTGTGCTAACTCGAG saposin $D^{\mathrm{w}}$ :

ATGGGCAGCAGCCATCATCATCATCATCACAGCAGCGGCCTGGTGCCGCGCGGCAGCCA TATGgGTTTTTGTGAAGTTTGCAAAAAGTTGgTTTTGTATTTGGAACATAACTTGGAAAAGA ACAGCACCAAAGAAGAAATTTTGGCCGCCTTGGAAAAAGGTTGTAGCTGGTTGCCGGATC 
CTTATCAAAAACAGTGCGATGATTTTGTTGCCGAATATGAACCGTTGTTGTTGGAAATTTTG GTTGAAGTTATGGATCCGGGCTTTGTTTGCTCTAAAATTGGTGTTTGTCCTAGCTAACTCGA G

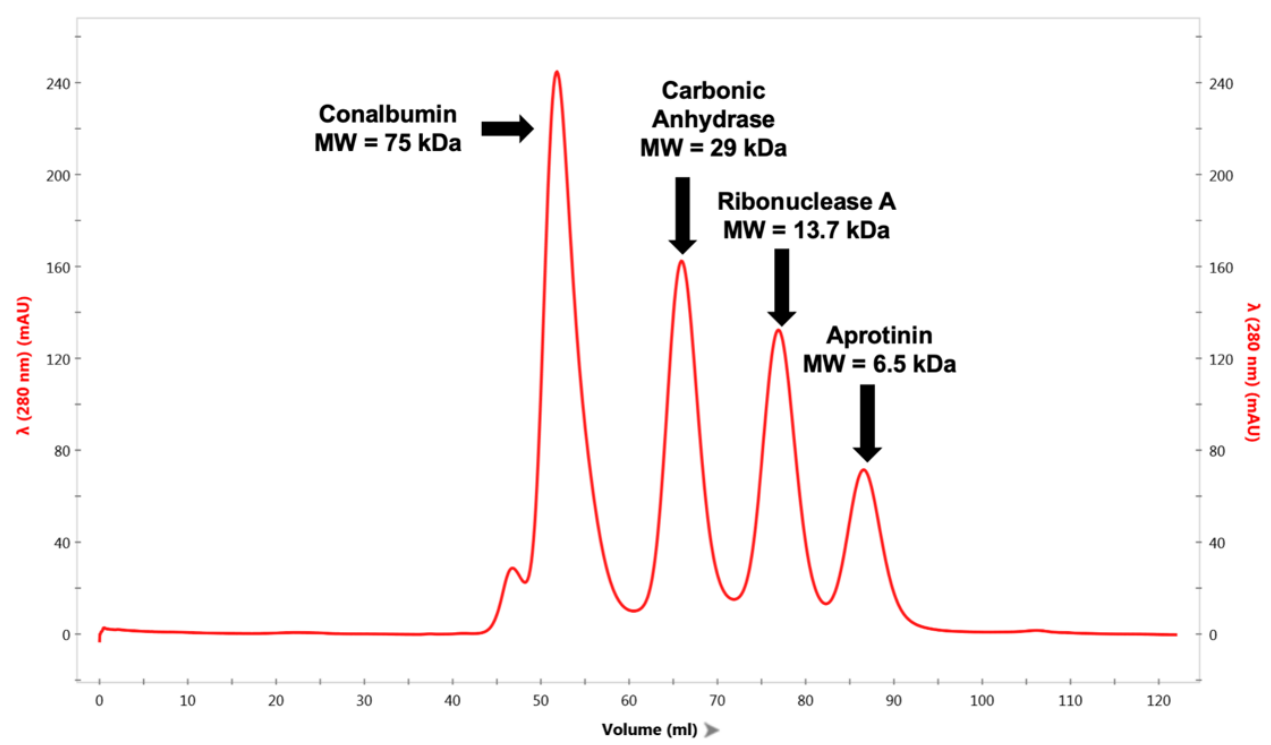

Figure S2. SEC elution profile (Superdex 75) of a sample including four molecular weight standards: conalbumin $(\mathrm{MW}=75 \mathrm{kDa})$, carbonic anhydrase $(\mathrm{MW}=29 \mathrm{kDa})$, ribonuclease $\mathrm{A}(\mathrm{MW}$ $=13.7 \mathrm{kDa})$, and aprotinin (MW = 6.5 kDa) (GE Lifesciences). The elution buffer used was $25 \mathrm{mM}$ Tris- $\mathrm{HCl} \mathrm{pH}$ 7.5, 100 mM Na2SO4, 2\% glycerol.

\section{Tryptophan Fluorescence Emission Spectroscopy:}
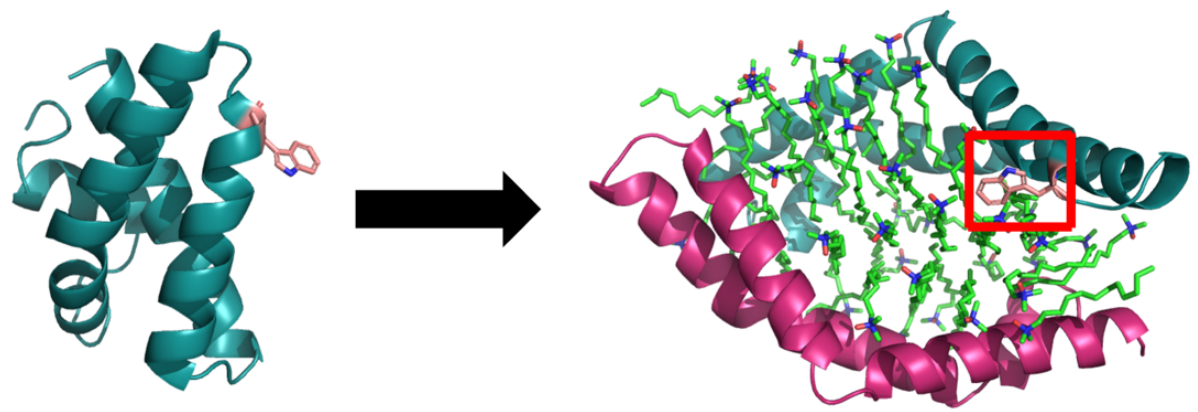
Figure S3. Structural basis for tryptophan fluorescence emission studies. Saposin A possesses a single tryptophan residue. The Trp side chain is oriented toward solution in the saposin A monomeric form (left, $\mathrm{PDB} 2 \mathrm{DOB}^{5}$ ), but the Trp side chain is oriented toward detergent in the picodisc assembly (PDB 4DDJ). This change in environment surrounding the Trp side chain leads to a blue shift and an intensity increase in the Trp fluorescence emission profile when the monomeric state is converted to a picodisc assembly.

Table S1. Detergents assessed for binding to saposin A.

\begin{tabular}{|c|c|c|}
\hline Name & $\mathrm{CMC}^{\mathrm{a}}$ & $\begin{array}{c}\text { Detergent } \\
\text { Conc }^{b} / \mathbf{C M C}^{c}\end{array}$ \\
\hline n-decyl- $\beta$-D-maltopyranoside & In water $=1.8 \mathrm{mM}$; in $0.15 \mathrm{M} \mathrm{NaCl}=1.8 \mathrm{mM}$ & $\sim 0.11$ \\
\hline n-dodecyl- $\beta$-D-maltopyranoside & In water $=0.17 \mathrm{mM}$; in $0.2 \mathrm{M} \mathrm{NaCl}=0.12 \mathrm{mM}$ & $\sim 1.7$ \\
\hline n-tridecyl- $\beta$-D-maltopyranoside & In water $=0.033 \mathrm{mM}$; in $0.15 \mathrm{M} \mathrm{NaCl}=0.024 \mathrm{mM}$ & $\sim 8.3$ \\
\hline n-tetradecyl- $\beta$-D-maltopyranoside & In water $\sim 0.01 \mathrm{mM}$ & $\sim 20$ \\
\hline MNA-C12 & In water $=4 \mu \mathrm{M}$ & $\sim 50$ \\
\hline MNA-C13 & In water $=2 \mu \mathrm{M}$ & $\sim 100$ \\
\hline lauryl maltoe neopentyl glycol (LMNG) & In water $\sim 0.01 \mathrm{mM}$ & $\sim 20$ \\
\hline n-octyl- $\beta$-D-thioglucopyranoside & In water $=8.5 \mathrm{mM}$ & $\sim 0.023$ \\
\hline n-octyl- $\beta$-D-glucopyranoside ( $\beta O G)$ & In water $=18-20 \mathrm{mM}$; in $0.1 \mathrm{M} \mathrm{NaCl}=23.4 \mathrm{mM}$ & $\sim 0.0085$ \\
\hline octyl glucose neopentyl glycol & In water $=1.02 \mathrm{mM}$ & $\sim 0.20$ \\
\hline CHAPS & In water $=8 \mathrm{mM}$ & $\sim 0.025$ \\
\hline $\begin{array}{l}\text { CHAPSO } \\
\text { VHed }\end{array}$ & In water $=8 \mathrm{mM}$ & $\sim 0.025$ \\
\hline $\begin{array}{l}\text { (LDAO) } \\
\text { (LDecyi-IN IN }\end{array}$ & In water $=1-2 \mathrm{mM}$; in $0.1 \mathrm{M} \mathrm{NaCl}=0.14 \mathrm{mM}$ & $\sim 1.4$ \\
\hline
\end{tabular}

${ }^{a}$ The listed CMC values were measured by Anatrace. ${ }^{b}$ The "detergent concentration" indicated in the rightmost heading refers to the concentration of detergent in the sample containing saposin A. "None of the detergents with $\mathrm{CMC}$ values below the detergent concentration used with saposin A formed a co-assembly with this protein. 
A.

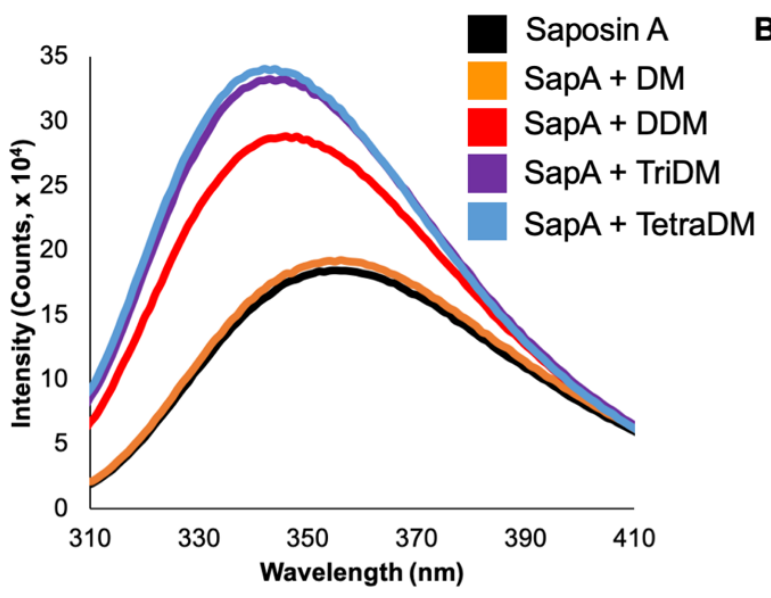

B.

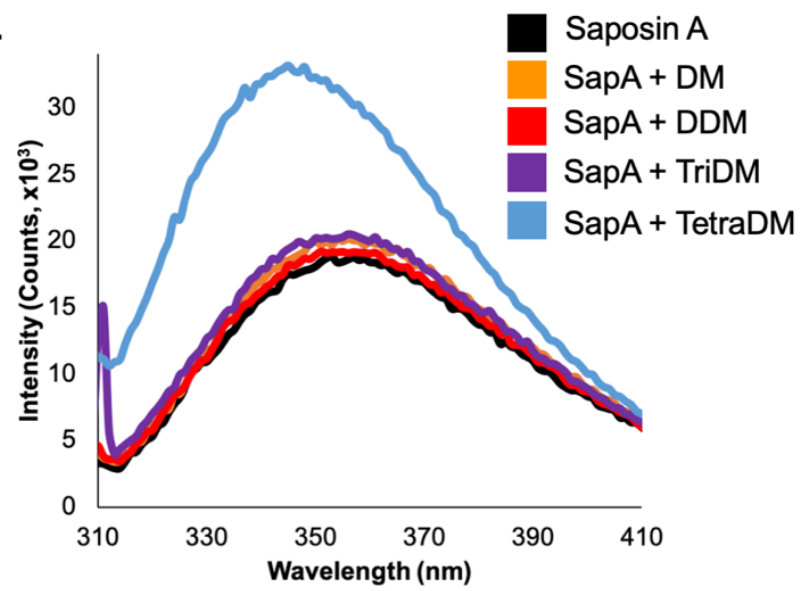

Figures S4. Tryptophan fluorescence emission spectra of monomeric saposin A (black) overlaid with data collected from samples of saposin $A$ incubated in a 1:10 ratio with detergent: (DM (orange), DDM (red), TriDM (purple) and TetraDM (blue)) [in $25 \mathrm{mM}$ Tris-HCl, pH 7.5, $150 \mathrm{mM}$ $\mathrm{NaCl}$ buffer conditions]. Panels A shows data for $20 \mu \mathrm{M}$ monomeric saposin A (black, for reference) and for $20 \mu \mathrm{M}$ saposin A combined with the indicated detergent at $200 \mu \mathrm{M}$. Panel B shows data for $2 \mu \mathrm{M}$ monomeric saposin A (black, for reference) and for $2 \mu \mathrm{M}$ saposin A combined with the indicated detergent at $20 \mu \mathrm{M}$.

A.

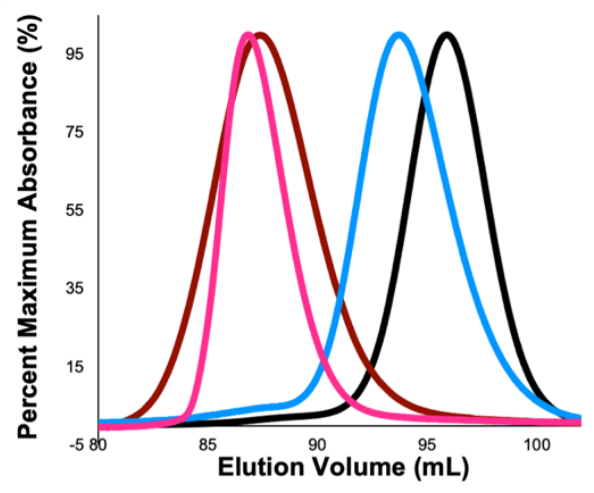

B.

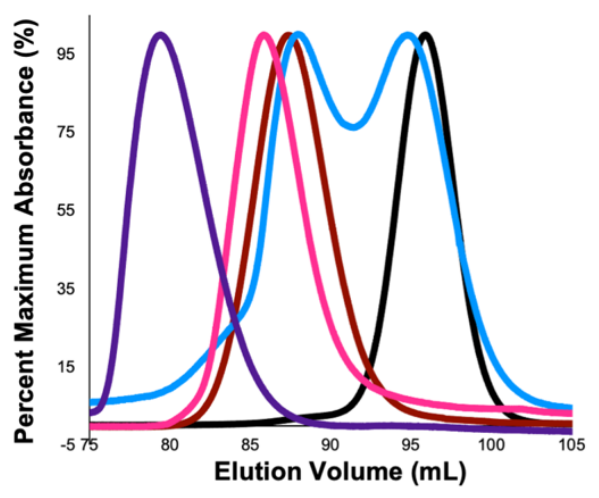

Monomeric SapA

1:10 SapA:Detergent

1:20 SapA:Detergent

1:40 SapA:Detergent

1:10 SapA:MNA C12

Figures S5. Effects of detergents TriDM and TetraDM on saposin A. SEC (Superdex 200) elution profiles of saposin A incubated with different protein:detergent ratios were compared with monomeric saposin A (black traces) to determine whether assemblies formed. Panel A shows the 
profiles for saposin A-TriDM co-assembly, and panel B shows the profiles for saposin A-TetraDM co-assembly. The colors of the traces indicate the protein:detergent ratio: green (1:5), blue (1:10), pink (1:20) and purple (1:40). The elution profile for 1:10 saposin A:MNA C12 is included as a reference for a saposin $A+$ detergent picodisc assembly. Comparison with this reference suggests that picodisc assemblies containing two saposin $A$ molecules are formed when saposin $A$ is combined with TriDM or Tetra DM in a 1:20 ratio. The elution buffer used was $25 \mathrm{mM}$ Tris- $\mathrm{HCl}$, $\mathrm{pH} 7.5,150 \mathrm{mM} \mathrm{NaCl}$.

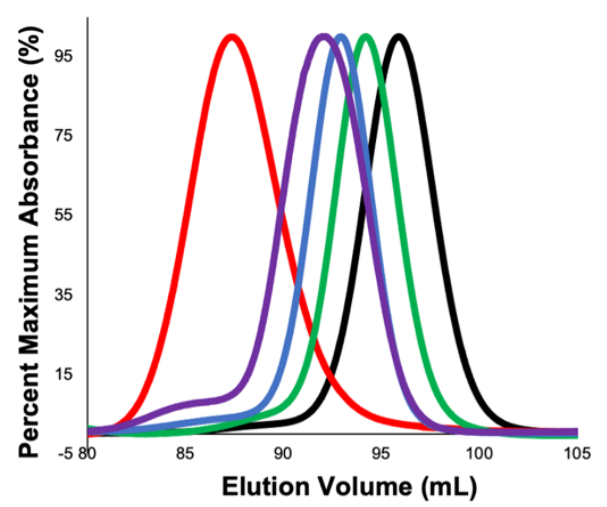

Monomeric SapA

1:37 SapA:Detergent

1:74 SapA:Detergent

1:148 SapA:Detergent

1:10 SapA:MNA C12

Figures S6. SEC elution profiles (Superdex 200) of monomeric saposin A (black) compared with 1: 37 saposin A:DDM (green), 1: 74 saposin A:DDM (blue), 1: 148 saposin A:DDM (purple), and 1:10 saposin A:MNA C12. In each sample the concentration of DDM (3.9 mM, $7.8 \mathrm{mM}$, and 15 $\mathrm{mM}$ respectively) exceeded the $\mathrm{CMC}$ value of this detergent $(\sim 0.17 \mathrm{mM})$. No saposin $\mathrm{A}+\mathrm{DDM}$ combination formed a species that was similar in size to a picodisc assembly involving two saposin A molecules along with detergent; this type of assembly is represented in the figure by the 1:10 saposin A:MNA C12 profile (red). The elution buffer used was $25 \mathrm{mM}$ Tris- $\mathrm{HCl}, \mathrm{pH} 7.5$, $150 \mathrm{mM} \mathrm{NaCl}$. 

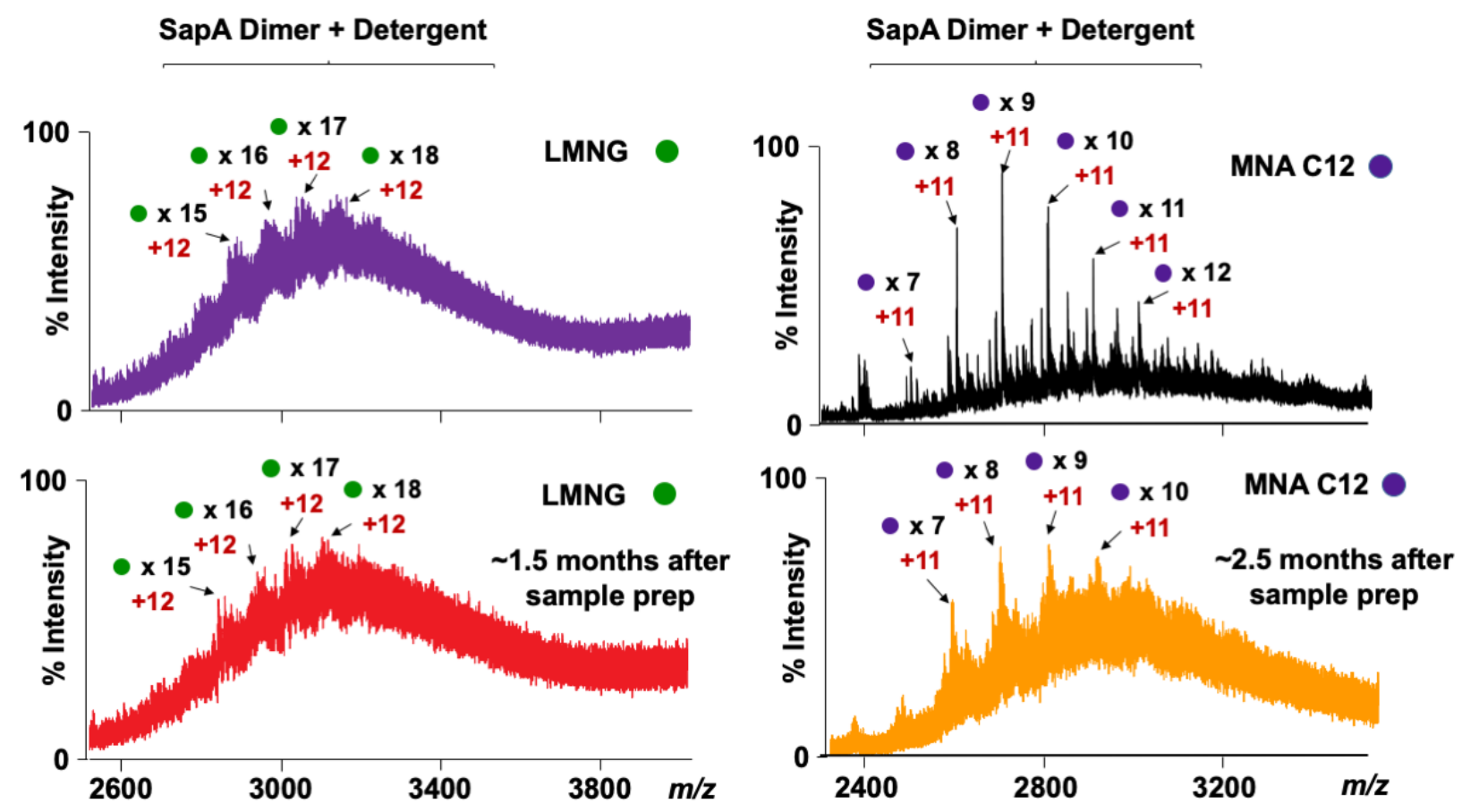

Figure S7. Native mass spectra of saposin A+detergent co-assemblies obtained 2-5 days after sample preparation (top) and several weeks after sample preparation (bottom). At the later time, the characteristic signals display lower intensity (which may indicate higher sample heterogeneity), but these comparisons show that picodisc assembly composition is maintained over multiple weeks. 


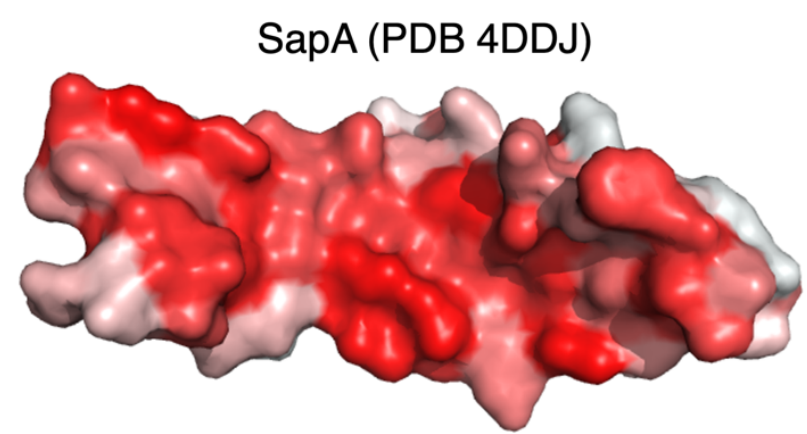

SapC (PDB 2Z9A)

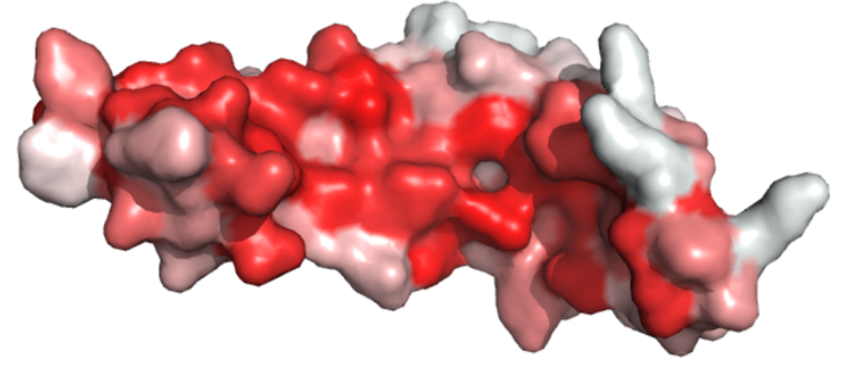

HYDROPHOBICITY

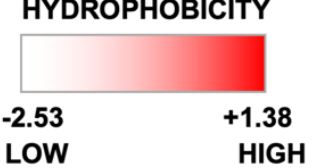

SapB (PDB 1N69)

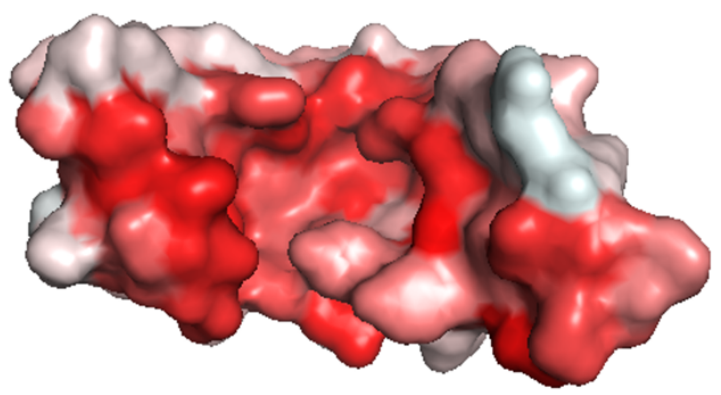

SapD (PDB 5U85)

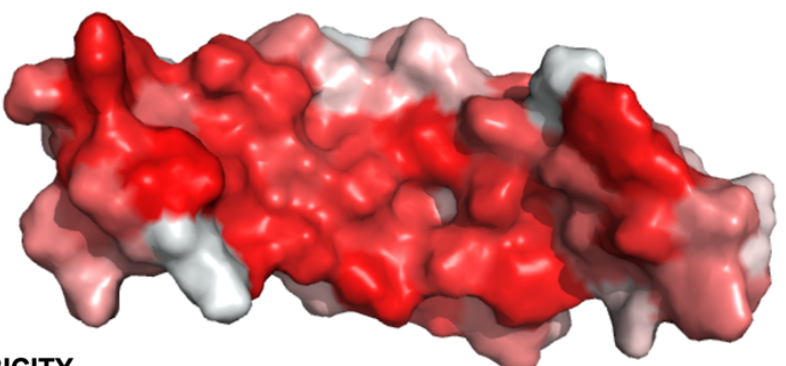

HIGH

Figures S8. Comparison of the hydrophobic surfaces displayed in the open conformation adopted by saposin $A$ in the saposin $A+L D A O$ structure (PDB 4DDJ) and the dimer conformations of $\operatorname{saposin} B, C$, and $D$. 
A.

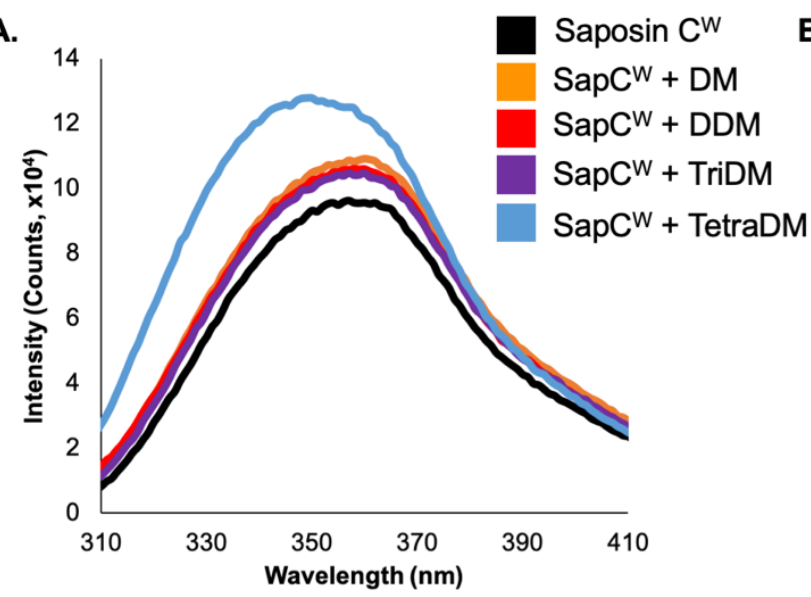

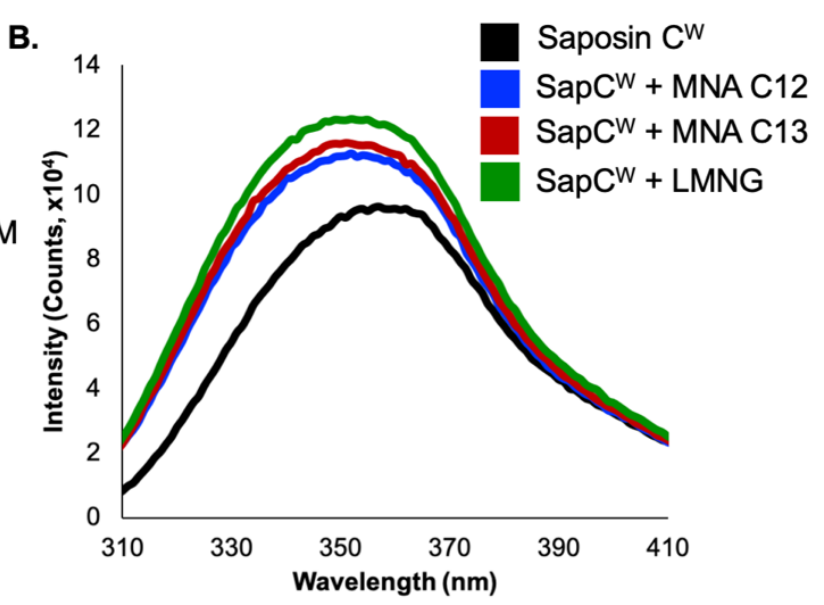

Figures S9. Tryptophan fluorescence emission spectra of saposin $C^{W}(2 \mu \mathrm{M}$, black) overlaid with data collected from samples of saposin $C^{W}(2 \mu \mathrm{M})$ incubated with detergent $(20 \mu \mathrm{M})$ in $25 \mathrm{mM}$ Tris- $\mathrm{HCl}, \mathrm{pH} 7.5,150 \mathrm{mM} \mathrm{NaCl}$. Panel A shows the emission spectra of saposin $\mathrm{C}^{\mathrm{W}}$ combined with detergent DM (orange), DDM (red), TriDM (purple) or TetraDM (blue). Panel B shows the emission spectra of saposin $\mathrm{C}^{\mathrm{W}}$ combined with MNA C12 (royal blue), MNA C13 (dark red) or LMNG (green).

A.

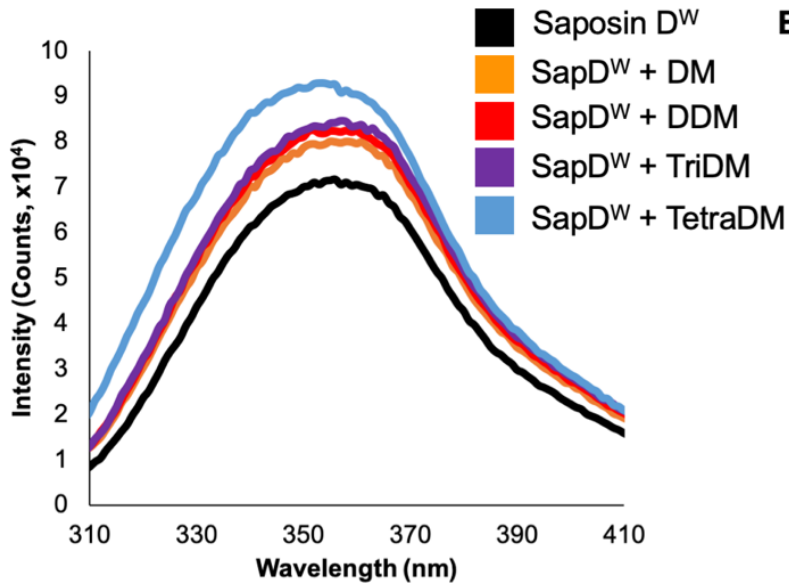

B.

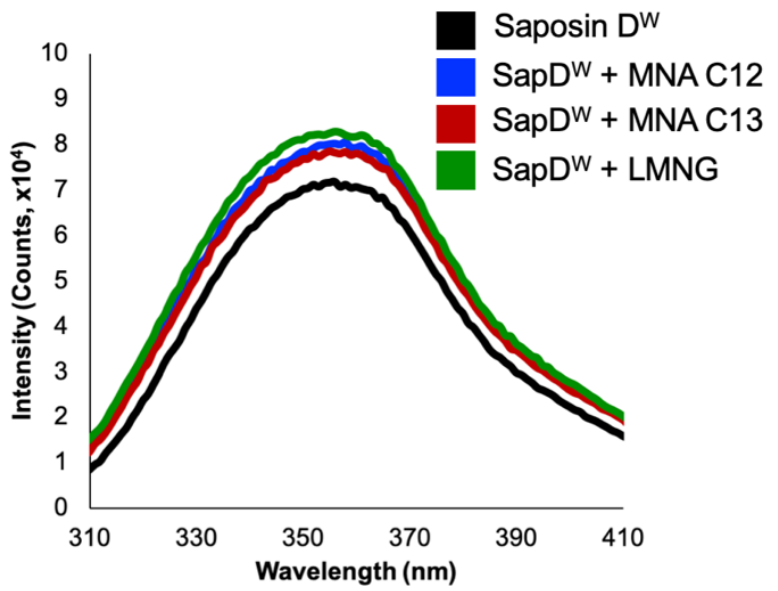

Figures S10. Tryptophan fluorescence emission spectra of saposin $D^{W}(2 \mu M$, black) overlaid with data collected from samples of saposin $D^{W}(2 \mu M)$ incubated with $20 \mu \mathrm{M}$ detergent in $25 \mathrm{mM}$ Tris- $\mathrm{HCl}, \mathrm{pH} 7.5,150 \mathrm{mM} \mathrm{NaCl}$. Panel A shows the emission spectra of saposin $\mathrm{D}^{\mathrm{W}}$ combined 
with detergent DM (orange), DDM (red), TriDM (purple) or TetraDM (blue). Panel B shows the emission spectra of saposin $D^{\mathrm{W}}$ combined with MNA C12 (royal blue), MNA C13 (dark red) or LMNG (green).
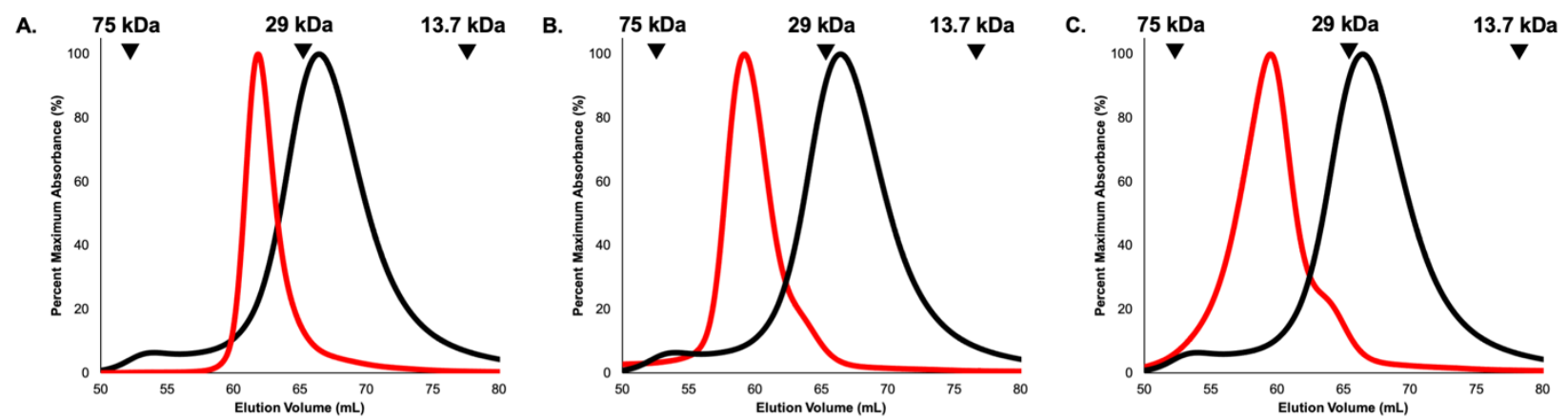

Figure S11. SEC chromatograms (Superdex 75) of saposin $\mathrm{B}^{\mathrm{W}}$ (black) overlaid with the following saposin $B^{W}+$ detergent combinations (red): 1:10 saposin $B^{W}: M N A C 12(A), 1: 10$ saposin $B^{W}: M N A$ C13 (B) and 1:15 saposin $B^{W}:$ LMNG $(C)$. For reference, the elution volumes of molecular weight standards are indicated by black triangles. The elution buffer used was $25 \mathrm{mM}$ Tris- $\mathrm{HCl}, \mathrm{pH} 7.5$, $150 \mathrm{mM} \mathrm{NaCl}$.

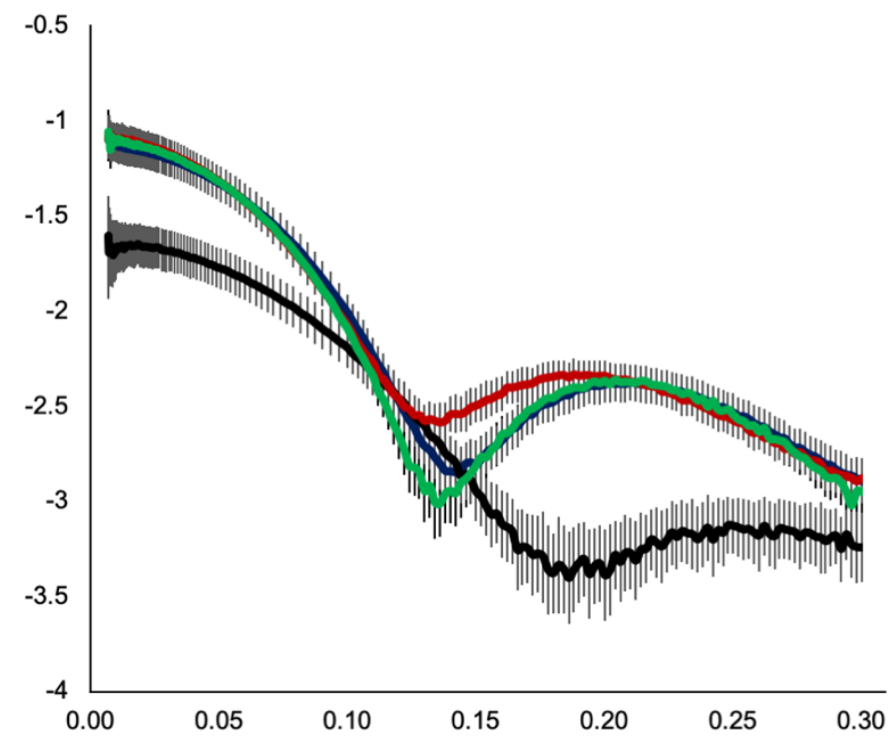

Figure S12. Overlay of experimental SEC-SAXS profiles of detergent-free dimeric saposin $\mathrm{B}^{\mathrm{W}}$ (black), saposin $B^{W}+M N A C 12$ (navy), saposin $B^{W}+M N A C 13$ (red), and saposin $B^{W}+$ LMNG (green). See below for experimental details. 
A.

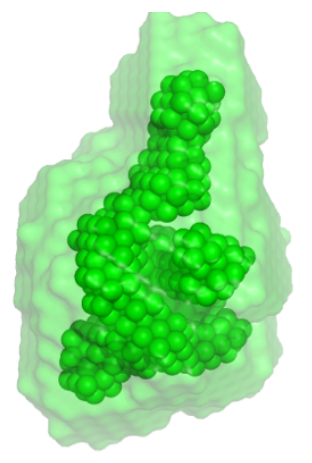

C.

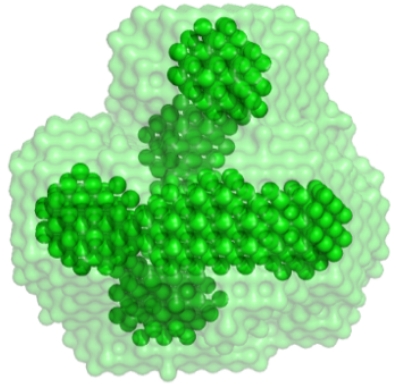

E.

G.
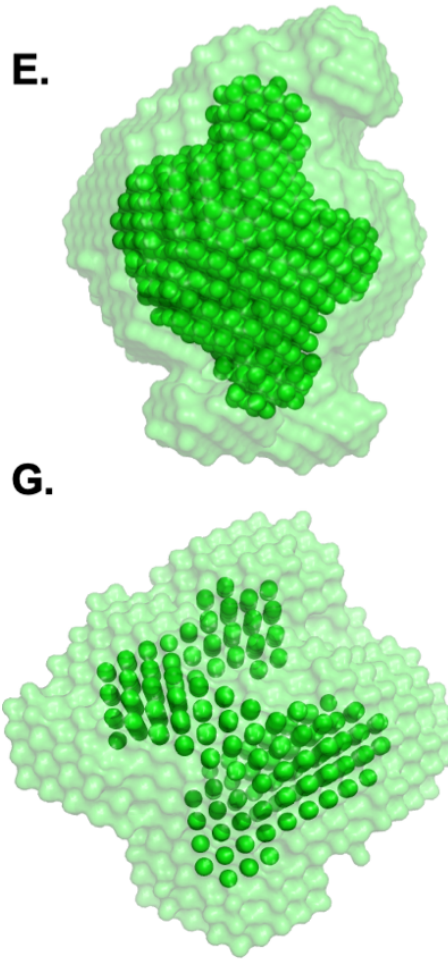
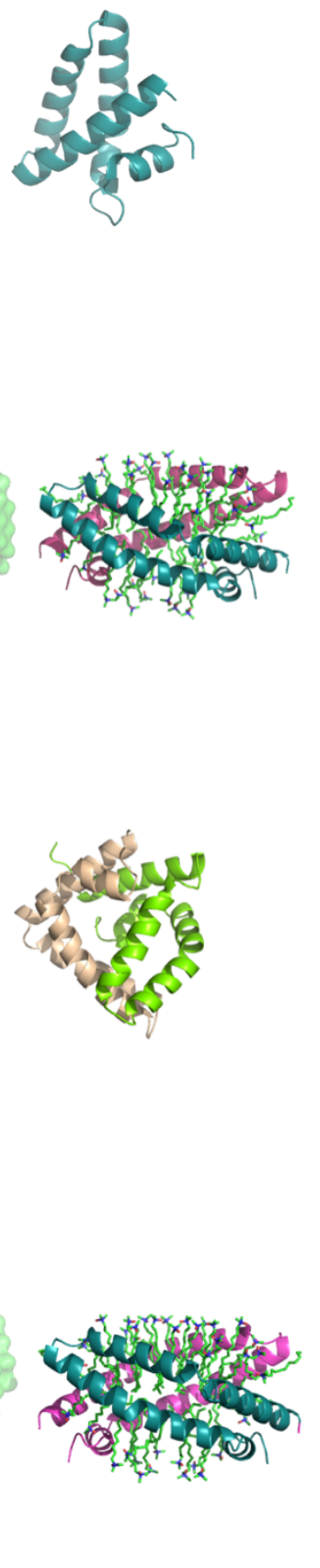

B.

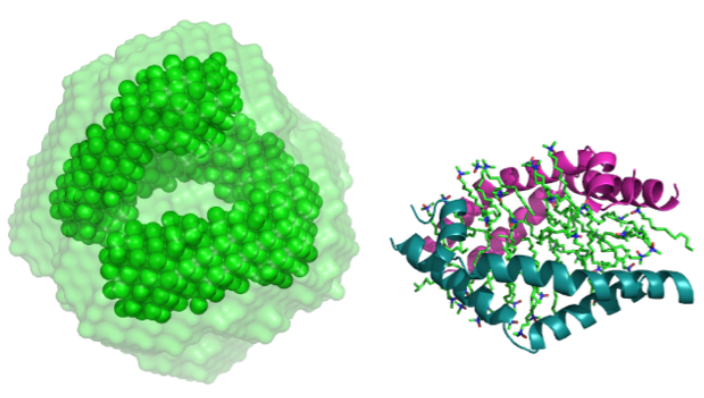

D.
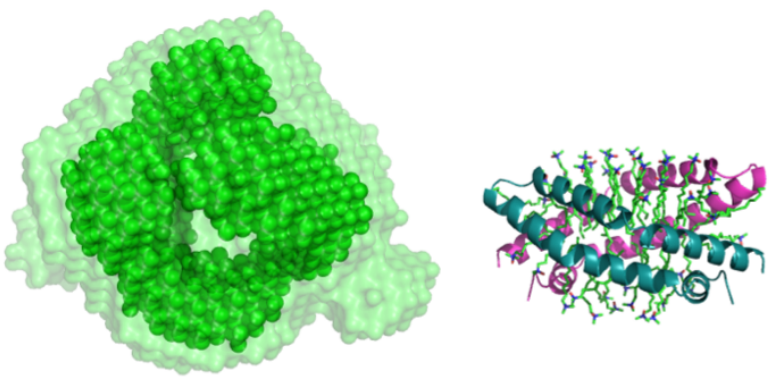

F.
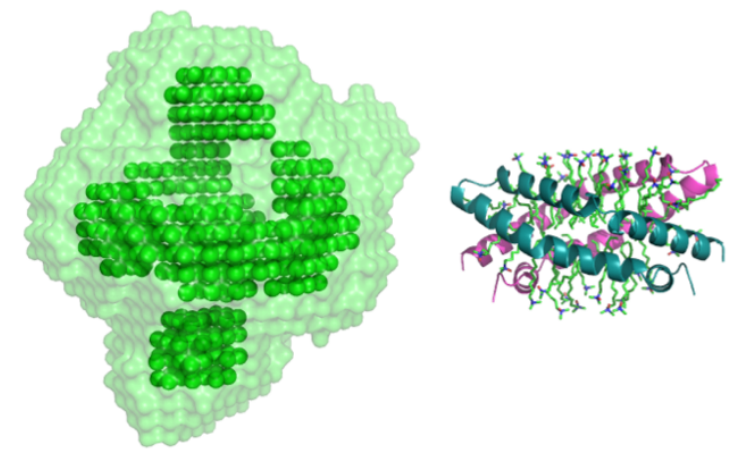

H.

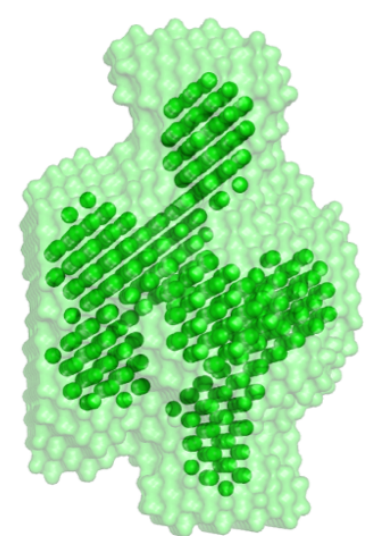

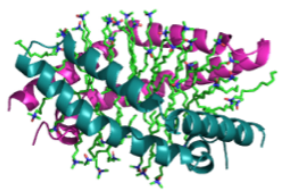

Figure S13. Ab initio models of monomeric saposin A (A), saposin A+MNA C12 (B), saposin $A+M N A C 13(C)$, saposin $A+L M N G(D)$, dimeric saposin $B^{W}(E)$, saposin $B^{W}+M N A C 12(F)$, saposin $B^{W}+M N A C 13(G)$, and saposin $B^{W}+L M N G(G)$ were generated based on each system's SEC-SAXS data using the program DAMMIF. ${ }^{6}$ For each SEC-SAXS dataset, twenty independent 
ab initio models, composed of "dummy atoms" or spherical volumes of electron density, were generated based on optimizing the alignment between the calculated SAXS signature of the model and that of the experimental data. These twenty models were then averaged via the software, DAMAVER; the averaged model of each analyzed system is shown here as a surface. A more compact model based on the DAMAVER models was acquired via DAMFILT, a program that identifies the dummy atoms most frequently present in the independent models. These models are shown here as spheres. For reference, we have included the crystal structures of the saposin A monomer (PDB 2DOB, panel A), the saposin A+LDAO picodisc (PDB 4DDJ, panels B$\mathrm{D}, \mathrm{F}-\mathrm{H})$, and the dimeric conformation of saposin B (PDB 1N69) in order to provide benchmarks for molecular size.

Table S2. Summary of the structural characterization of the identified saposin A+detergent and saposin $\mathrm{B}^{\mathrm{W}}+$ detergent picodisc assemblies

\begin{tabular}{ccc}
\hline System & $\begin{array}{c}\text { Sap:Det Ratio } \\
\text { (Native MS) }^{a}\end{array}$ & $\begin{array}{c}\text { Rg }(\AA) \\
\text { (SEC-SAXS) }^{b}\end{array}$ \\
\hline SapA+triDM & NA & \\
SapA+tetraDM & NA & \\
SapA+MNA C12 & $2: 7-12$ & $22.8+/-2.0$ \\
SapA+MNA C13 & $2: 8-14$ & $25.2+/-3.4$ \\
SapA+LMNG & $2: 15-18$ & $24.9+/-1.4$ \\
SapBW+MNA C12 & & $23.9+/-2.5$ \\
SapBW+MNA C13 & & $25.5+/-2.1$ \\
SapBW+LMNG & & $27.7+/-3.6$ \\
SapA monomer & NA & $15.4+/-1.4$ \\
SapBW dimer & NA & $18.9+/-2.2$
\end{tabular}

${ }^{a}$ The number of detergent molecules per picdisc was quantified by native mass spectrometry. ${ }^{b}$ The radii of gyration were determined by Guinier analysis using the RAW platform (see below for details). ${ }^{7}$ 
Table S3. Data acquisition parameters, sample details, data analysis and modelling fitting for the SEC-SAXS data collection and analysis of saposin $A$, saposin $A+$ detergent assemblies, saposin $\mathrm{B}^{\mathrm{W}}$, and $\operatorname{saposin} \mathrm{B}^{\mathrm{W}}+$ detergent assemblies. The format of the following table was adopted from Trewhella, J., et al. ${ }^{8}$

\begin{tabular}{|c|c|c|c|c|c|c|c|c|}
\hline \multicolumn{9}{|l|}{ (a) Sample details } \\
\hline \multirow{6}{*}{$\begin{array}{l}\text { Organism } \\
\text { Expression System } \\
\text { Extinction coefficient } \varepsilon^{9} \\
\text { (wavelength and units) }\end{array}$} & $\begin{array}{l}\text { saposin } \\
\text { A }\end{array}$ & $\begin{array}{l}\text { saposin } \\
\text { A + MNA } \\
\text { C12 }\end{array}$ & $\begin{array}{l}\text { saposin } \\
\text { A + MNA } \\
\text { C13 }\end{array}$ & $\begin{array}{l}\text { saposin } \\
\text { A }+ \\
\text { LMNG }\end{array}$ & $\begin{array}{l}\text { saposin } \\
\mathrm{B}^{\mathrm{W}}\end{array}$ & $\begin{array}{l}\text { saposin } \\
B^{W}+ \\
\text { MNA C12 }\end{array}$ & $\begin{array}{l}\text { saposin } \\
B^{W}+ \\
\text { MNA C13 }\end{array}$ & $\begin{array}{l}\text { saposin } \\
\text { B }^{\mathrm{W}}+ \\
\text { LMNG }\end{array}$ \\
\hline & $\begin{array}{l}H . \\
\text { sapiens }\end{array}$ & $\begin{array}{l}H . \\
\text { sapiens }\end{array}$ & $\begin{array}{l}H . \\
\text { sapiens }\end{array}$ & $\begin{array}{l}H . \\
\text { saniens }\end{array}$ & $\begin{array}{l}H . \\
\text { saniens }\end{array}$ & $\begin{array}{l}H . \\
\text { saniens }\end{array}$ & $\begin{array}{l}H . \\
\text { saniens }\end{array}$ & $\begin{array}{l}H . \\
\text { saniens }\end{array}$ \\
\hline & E. coli & E. coli & E. coli & E. coli & E. coli & E. coli & E. coli & E. coli \\
\hline & $\begin{array}{l}9005 \mathrm{M}^{-} \\
{ }^{1} \mathrm{~cm}^{-1} \text { in } 6\end{array}$ & $\begin{array}{l}9005 \mathrm{M}^{-} \\
{ }^{1} \mathrm{~cm}^{-1} \text { in } 6\end{array}$ & $\begin{array}{l}9005 \mathrm{M}^{-} \\
{ }^{1} \mathrm{~cm}^{-1} \text { in } 6\end{array}$ & $\begin{array}{l}9005 \mathrm{M}^{-} \\
{ }^{1} \mathrm{~cm}^{-1} \text { in } 6\end{array}$ & $\begin{array}{l}9005 \mathrm{M}^{-} \\
{ }^{1} \mathrm{~cm}^{-1} \text { in } 6\end{array}$ & $\begin{array}{l}9005 \mathrm{M}^{-} \\
{ }^{1} \mathrm{~cm}^{-1} \text { in } 6\end{array}$ & $\begin{array}{l}9005 \mathrm{M}^{-} \\
{ }^{1} \mathrm{~cm}^{-1} \text { in } 6\end{array}$ & $\begin{array}{l}9005 \mathrm{M}^{-} \\
{ }^{1} \mathrm{~cm}^{-1} \text { in } 6\end{array}$ \\
\hline & & $M$ & $M$ & $\mathrm{M}$ & $M$ & $M$ & $M$ & $M$ \\
\hline & $\begin{array}{l}\lambda=280 \\
\mathrm{~nm}\end{array}$ & $\begin{array}{l}\lambda=280 \\
\mathrm{~nm}\end{array}$ & $\begin{array}{l}\lambda=280 \\
\mathrm{~nm}\end{array}$ & $\begin{array}{l}\lambda=280 \\
\mathrm{~nm}\end{array}$ & $\begin{array}{l}\lambda=280 \\
\mathrm{~nm}\end{array}$ & $\begin{array}{l}\lambda=280 \\
\mathrm{~nm}\end{array}$ & $\begin{array}{l}\lambda=280 \\
\mathrm{~nm}\end{array}$ & $\begin{array}{l}\lambda=280 \\
\mathrm{~nm}\end{array}$ \\
\hline \multirow[t]{3}{*}{$\begin{array}{l}\text { Molecular mass } M \text { from chemical } \\
\text { composition (Da) }\end{array}$} & $\begin{array}{l}\text { saposin } \\
\text { A } \\
\text { monomer }\end{array}$ & $\begin{array}{l}\text { saposin } \\
\text { A dimer = } \\
1904252\end{array}$ & $\begin{array}{l}\text { saposin } \\
\text { A dimer = } \\
1904252\end{array}$ & $\begin{array}{l}\text { saposin } \\
\text { A dimer = } \\
1904252\end{array}$ & $\begin{array}{l}\text { saposin } \\
\text { B dimer = } \\
18377.84\end{array}$ & $\begin{array}{l}\text { saposin } \\
\text { B dimer = } \\
18377.84\end{array}$ & $\begin{array}{l}\text { saposin B } \\
\text { dimer }= \\
18377.84\end{array}$ & $\begin{array}{l}\text { saposin B } \\
\text { dimer }= \\
18377.84\end{array}$ \\
\hline & & MNA C12 & MNA C13 & LMNG = & & MNA C12 & , MNA & LMNG = \\
\hline & 9521.26 & $\begin{array}{l}= \\
1167.37\end{array}$ & $\begin{array}{l}= \\
1223.48\end{array}$ & 1005.19 & & $\begin{array}{l}= \\
1167.37\end{array}$ & $\begin{array}{l}C 13= \\
1223.48\end{array}$ & 1005.19 \\
\hline $\begin{array}{l}\text { For SEC-SAS, loading } \\
\text { volume/concentration, }\left(\mathrm{mg} \mathrm{ml}^{-1}\right)\end{array}$ & $\begin{array}{l}7 \mathrm{mg} / \mathrm{mL} \\
100 \mu \mathrm{L}\end{array}$ & $\begin{array}{l}7 \mathrm{mg} / \mathrm{mL} \\
100 \mu \mathrm{L}\end{array}$ & $\begin{array}{l}7 \mathrm{mg} / \mathrm{mL} \\
100 \mu \mathrm{L}\end{array}$ & $\begin{array}{l}7 \mathrm{mg} / \mathrm{mL} \\
100 \mu \mathrm{L}\end{array}$ & $\begin{array}{l}10 \\
\mathrm{mg} / \mathrm{mL}\end{array}$ & $\begin{array}{l}10 \\
\mathrm{mg} / \mathrm{mL}\end{array}$ & $\begin{array}{l}10 \\
\mathrm{mg} / \mathrm{mL}\end{array}$ & $\begin{array}{l}10 \\
\mathrm{mg} / \mathrm{mL}\end{array}$ \\
\hline injection volume $(\mu \mathrm{l})$, flow rate $(\mathrm{ml}$ & 0.2 & 0.2 & 0.2 & 0.2 & $100 \mu \mathrm{L}$ & $100 \mu \mathrm{L}$ & $100 \mu \mathrm{L}$ & $100 \mu \mathrm{L}$ \\
\hline $\left.\min ^{-1}\right)$ & & $\mathrm{mL} / \mathrm{min}$ & $\mathrm{mL} / \mathrm{min}$ & $\mathrm{mL} / \mathrm{min}$ & $\begin{array}{l}0.2 \\
\mathrm{~mL} / \mathrm{min}\end{array}$ & $\begin{array}{l}0.2 \\
\mathrm{~mL} / \mathrm{min}\end{array}$ & $\begin{array}{l}0.2 \\
\mathrm{~mL} / \mathrm{min}\end{array}$ & $\begin{array}{l}0.2 \\
\mathrm{~mL} / \mathrm{min}\end{array}$ \\
\hline \multicolumn{9}{|l|}{$\begin{array}{l}\text { Concentration (range/values) } \\
\text { measured and method }\end{array}$} \\
\hline \multirow[t]{2}{*}{ Solvent composition and source } & $25 \mathrm{mM}$ & $25 \mathrm{mM}$ & $25 \mathrm{mM}$ & $25 \mathrm{mM}$ & $25 \mathrm{mM}$ & $25 \mathrm{mM}$ & $25 \mathrm{mM}$ & $25 \mathrm{mM}$ \\
\hline & $\begin{array}{l}\text { Tris- } \mathrm{HCl} \\
\mathrm{pH} 7.5\end{array}$ & $\begin{array}{l}\text { Tris- } \mathrm{HCl} \\
\mathrm{pH} 7.5\end{array}$ & $\begin{array}{l}\text { Tris- } \mathrm{HCl} \\
\mathrm{pH} 7.5\end{array}$ & $\begin{array}{l}\text { Tris- } \mathrm{HCl} \\
\mathrm{pH} 7.5\end{array}$ & $\begin{array}{l}\text { Tris- } \mathrm{HCl} \\
\mathrm{pH} 7.5\end{array}$ & $\begin{array}{l}\text { Tris- } \mathrm{HCl} \\
\mathrm{pH} 7.5\end{array}$ & $\begin{array}{l}\text { Tris- } \mathrm{HCl} \\
\mathrm{pH} 7.5\end{array}$ & $\begin{array}{l}\text { Tris- } \mathrm{HCl} \\
\mathrm{pH} 7.5\end{array}$ \\
\hline
\end{tabular}




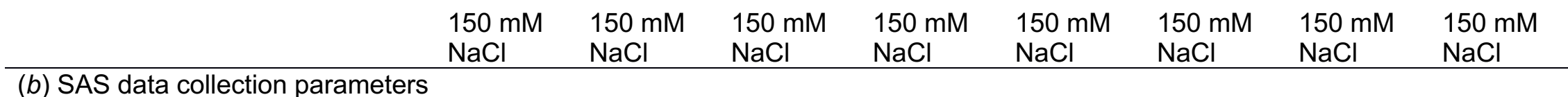

Source: APS Beamline 12-ID-B, Argonne National Lab

Wavelength: $0.9322 \AA$

Beam geometry: beam size $=0.10 \times 0.15 \mathrm{~mm}$, sample to detector distance

$=2.0 \mathrm{~m}$

q-measurement range : $0.005-0.8 \AA^{-1}$

Absolute scaling method: water as secondary standard

Exposure time: data taken every other second, 1 second / image

Sample path length: $1.5 \mathrm{~mm}$

Sample flow rate: $0.2 \mathrm{~mL} / \mathrm{min}$

Sample temperature: $25^{\circ} \mathrm{C}$

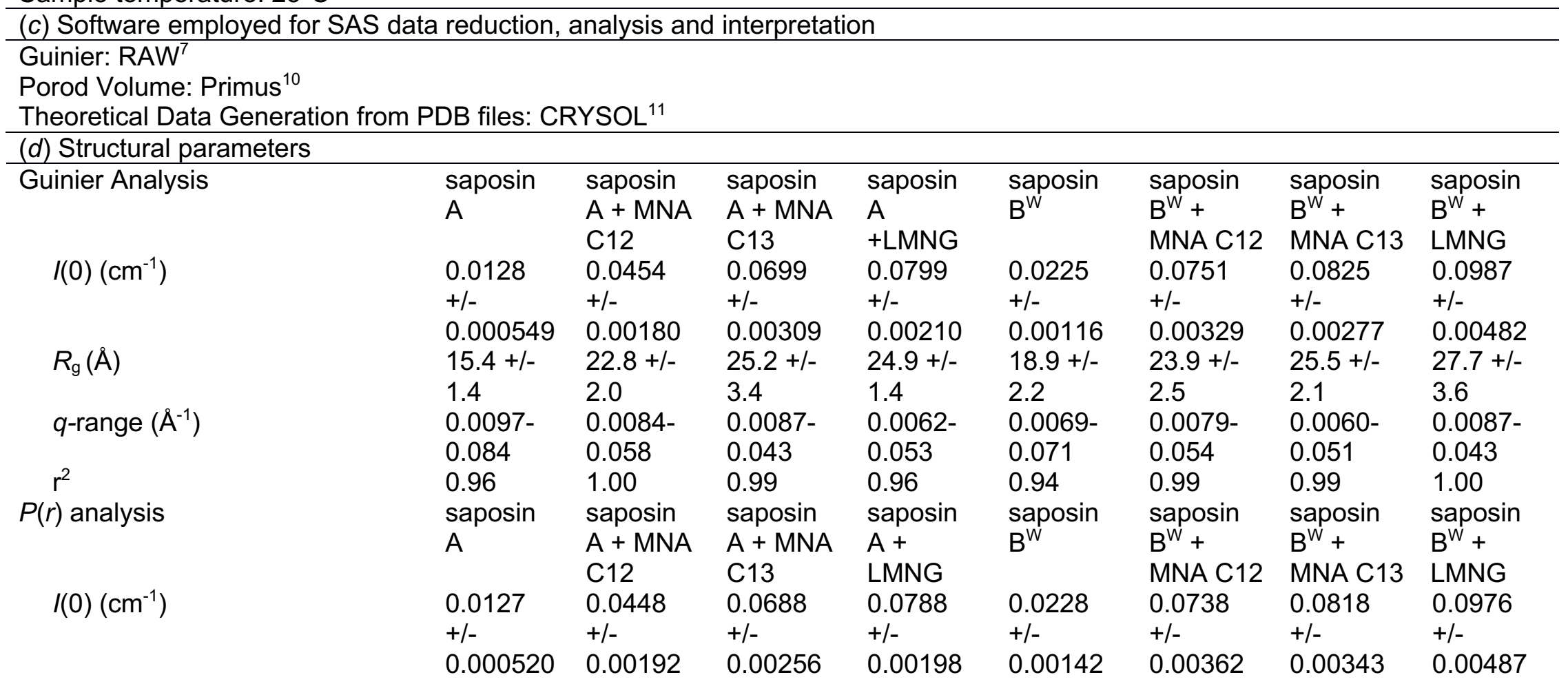




$\begin{array}{lllllllll}R_{g}(\AA) & 15.4+/- & 22.0+/- & 24.3+/- & 24.2+/- & 19.2+/- & 23.0+/- & 25.0+/- & 27.0+/- \\ & 0.7 & 0.6 & 0.7 & 0.6 & 1.0 & 1.2 & 1.2 & 2.0 \\ d_{\max }(\AA) & 49.5 & 62.0 & 67.0 & 69.7 & 57.6 & 69.2 & 77.1 & 89.2 \\ q \text {-range }\left(\AA^{-1}\right) & 0.013- & 0.0079- & 0.012- & 0.0093- & 0.018- & 0.014- & 0.0087- & 0.011- \\ & 0.52 & 0.35 & 0.32 & 0.32 & 0.41 & 0.34 & 0.31 & 0.29 \\ \text { Total Quality Estimate } & 0.82 & 0.81 & 0.82 & 0.80 & 0.82 & 0.76 & 0.70 & 0.63 \\ \text { Porod Volume } & 22800.00 & 19800.00 & 23300.00 & 24100.00 & 24700.00 & 21100.00 & 23700.00 & 31800.00\end{array}$

(e) CRYSOL Model Fit with

Saposin A Monomer (PDB 2DOB)

\begin{tabular}{|c|c|c|c|c|c|c|c|c|}
\hline & A & $\begin{array}{l}\text { saposin } \\
A+M N A \\
\text { C12 }\end{array}$ & $\begin{array}{l}\text { saposin } \\
A+\text { MNA } \\
\text { C13 }\end{array}$ & $\begin{array}{l}\text { saposin } \\
\text { A + } \\
\text { LMNG }\end{array}$ & $\begin{array}{l}\text { saposin } \\
\mathrm{B}^{\mathrm{W}}\end{array}$ & $\begin{array}{l}\text { saposin } \\
\mathrm{B}^{\mathrm{W}}+ \\
\text { MNA C12 }\end{array}$ & $\begin{array}{l}\text { saposin } \\
B^{W}+ \\
\text { MNA C13 }\end{array}$ & $\begin{array}{l}\text { saposin } \\
\mathrm{B}^{\mathrm{W}}+ \\
\text { LMNG }\end{array}$ \\
\hline$q$-range for fitting & $0-0.30$ & $0-0.30$ & $0-0.30$ & $0-0.30$ & & & & \\
\hline$R_{\mathrm{g}}$ of Theoretical Curve $(\AA)$ & 14.1 & 15.0 & 15.0 & 15.0 & & & & \\
\hline Optimal Excluded Volume $\left(\AA^{3}\right)$ & 11986 & 11986 & 11986 & 11986 & & & & \\
\hline $\begin{array}{l}\chi^{2} \text { value (discrepancy between } \\
\text { theoretical and Experimental } \\
\text { curves) }\end{array}$ & 0.04 & 8.29 & 10.52 & 20.71 & & & & \\
\hline \multicolumn{9}{|l|}{$\begin{array}{l}\text { (f) CRYSOL Model Fit with } \\
\text { Saposin B Dimer (PDB 1N69) }\end{array}$} \\
\hline & $\begin{array}{l}\text { saposin } \\
\text { A }\end{array}$ & $\begin{array}{l}\text { saposin } \\
\text { A + MNA } \\
\text { C12 }\end{array}$ & $\begin{array}{l}\text { saposin } \\
\text { A + MNA } \\
\text { C13 }\end{array}$ & $\begin{array}{l}\text { saposin } \\
\text { A + } \\
\text { LMNG }\end{array}$ & $\begin{array}{l}\text { saposin } \\
\mathrm{B}^{\mathrm{W}}\end{array}$ & $\begin{array}{l}\text { saposin } \\
B^{W}+ \\
\text { MNA C12 }\end{array}$ & $\begin{array}{l}\text { saposin } \\
B^{W}+ \\
\text { MNA C13 }\end{array}$ & $\begin{array}{l}\text { saposin } \\
\mathrm{B}^{\mathrm{W}}+ \\
\text { LMNG }^{\text {LMNG }}\end{array}$ \\
\hline q-range for fitting & & & & & $0-0.30$ & $0-0.30$ & $0-0.30$ & $0-0.30$ \\
\hline$R_{\mathrm{g}}$ of Theoretical Curve $(\AA)$ & & & & & 21.0 & 23.1 & 23.0 & 23.1 \\
\hline Optimal Excluded Volume $\left(\AA^{3}\right)$ & & & & & 35876 & 35876 & 35876 & 35876 \\
\hline $\begin{array}{l}\chi^{2} \text { value (discrepancy between } \\
\text { theoretical and Experimental } \\
\text { curves) }\end{array}$ & & & & & 0.21 & 4.17 & 5.59 & 6.08 \\
\hline $\begin{array}{l}\text { (g) CRYSOL Model Fit with } \\
\text { Saposin A+LDAO Disc (PDB } \\
\text { 4DDJ) }\end{array}$ & & & & & & & & \\
\hline
\end{tabular}




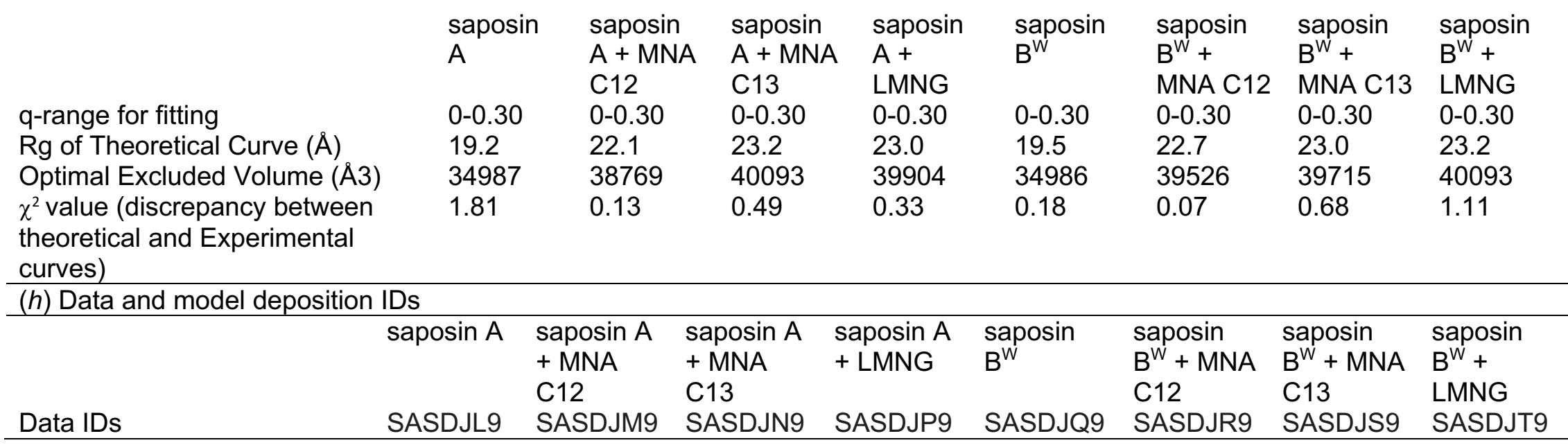


A.

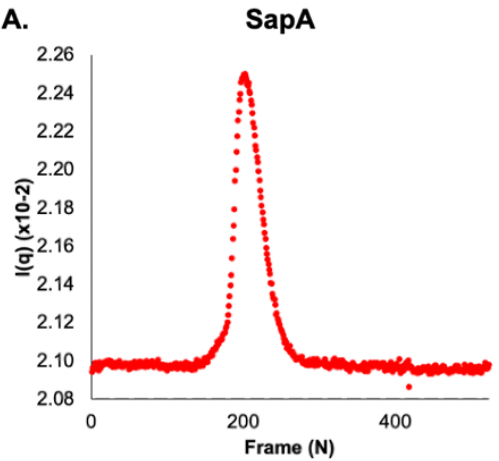

D.

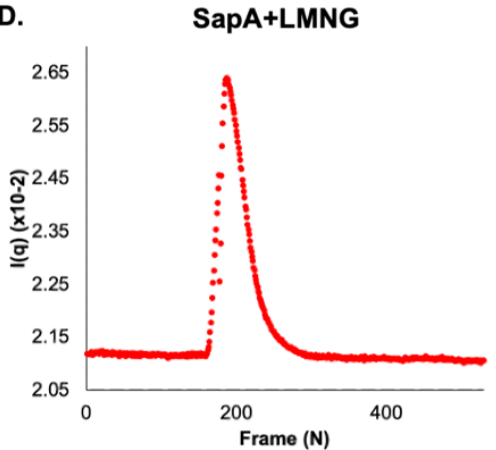

G.

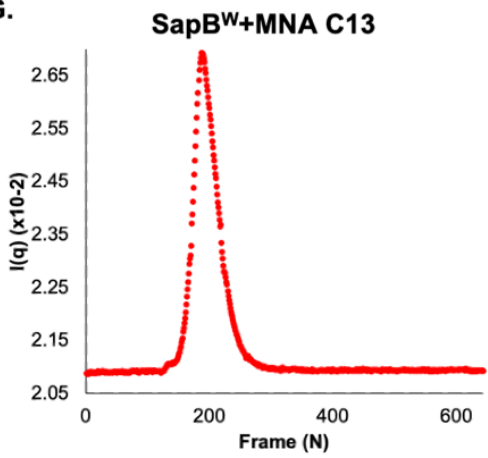

B.

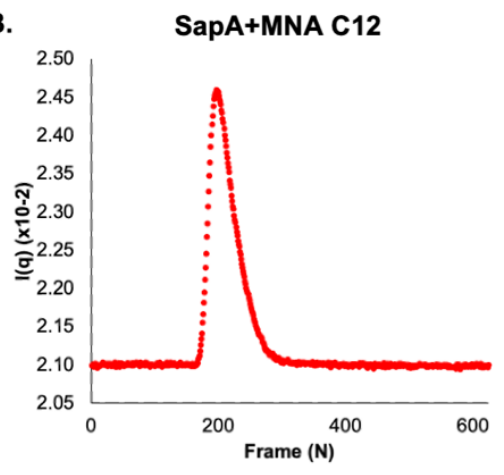

E.

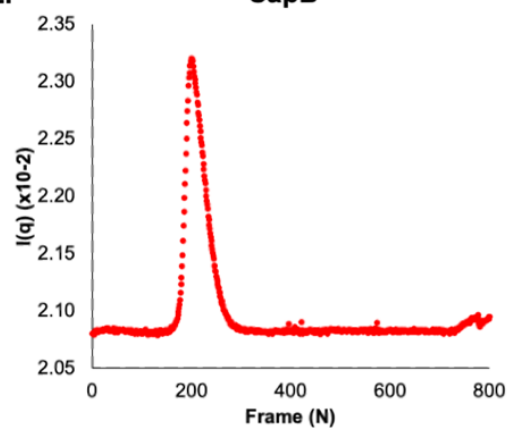

H.

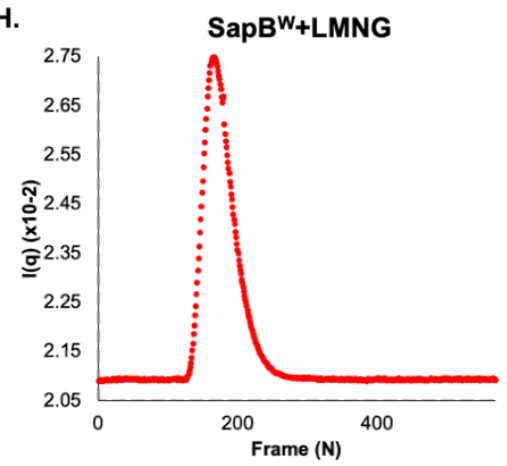

c.

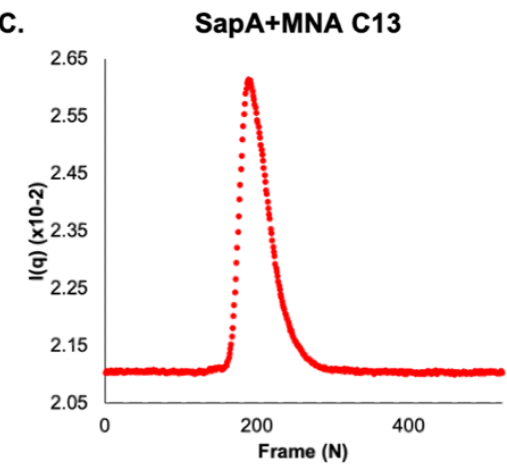

F.

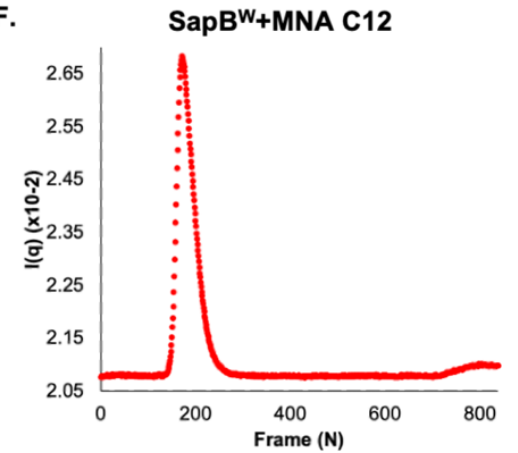

Figure S14. Plots of x-ray diffraction intensity per frame collected for each SEC-SAXS sample: saposin $A(A)$, saposin $A+M N A C 12(B)$, saposin $A+M N A C 13(C)$, saposin $A+L M N G(D)$, saposin $B^{W}(E)$, saposin $B^{W}+M N A C 12(F)$, saposin $B^{W}+M N A C 13(G)$, and saposin $B^{W}+L M N G(H)$. SAXS data were collected every other second at a rate of 1 second per frame. The intensity (I(q)) of each frame was plotted to determine which frames to merge to generate the final data set. Only the 10-20 frames with the highest I(q) values were merged. 
A.

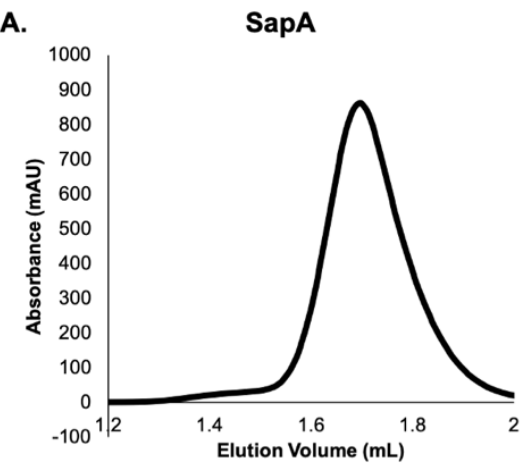

D.

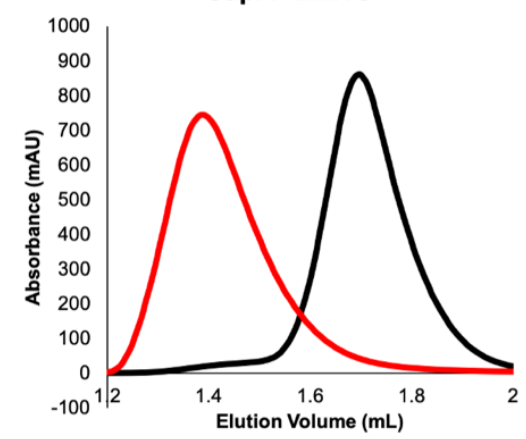

G.

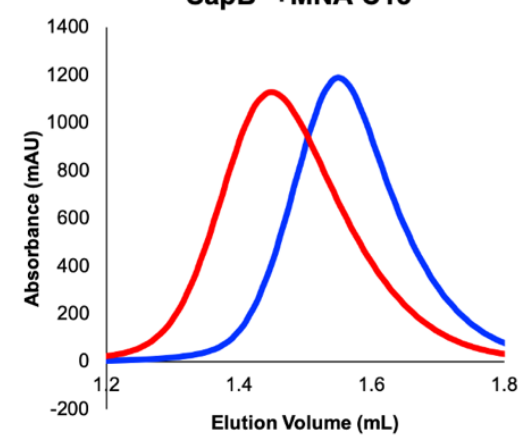

B.

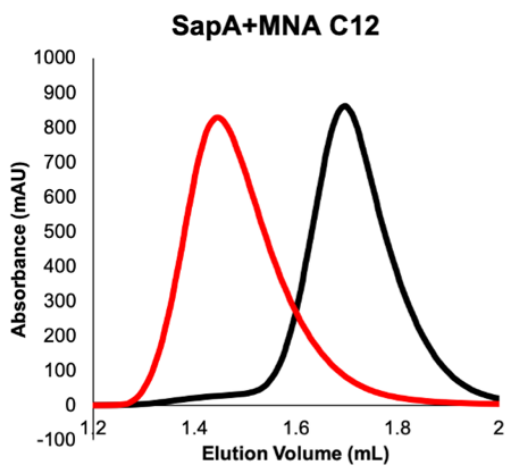

E.

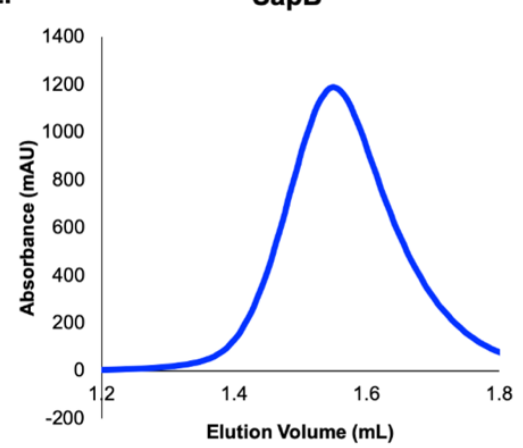

H.

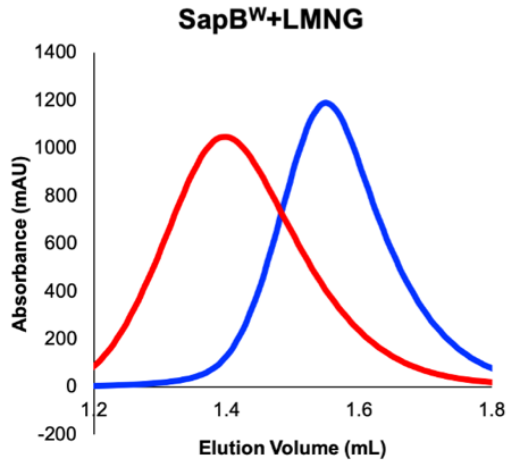

C. SapA+MNA C13

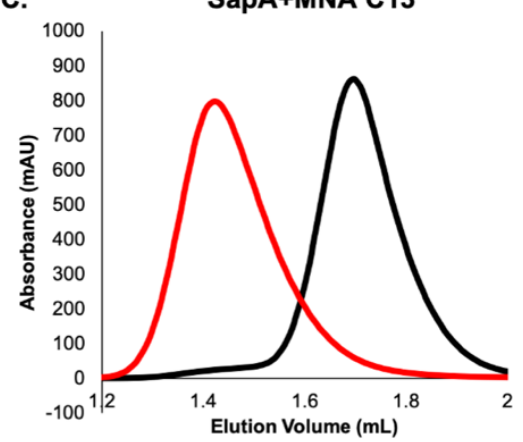

F.

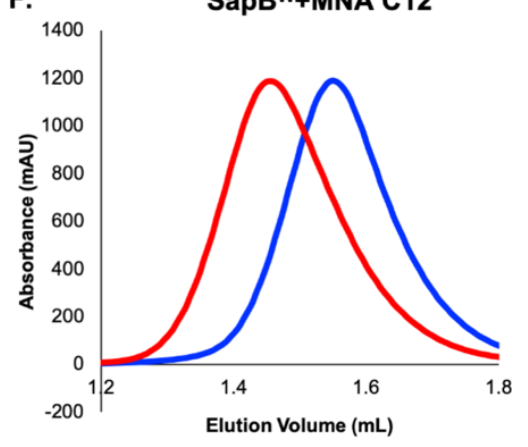

Figure S15. SEC (Superdex 75) elution profiles of the SEC-SAXS samples. Panel A shows the elution profile of monomeric saposin A. In panels B-D the profiles of the saposinA-detergent coassemblies are shown in red while the chromatogram of monomeric saposin A is shown in black for comparison. Panel $\mathrm{E}$ shows the elution profile of dimeric saposin $\mathrm{B}^{\mathrm{W}}$ (no detergent). In panels $\mathrm{F}-\mathrm{H}$, the elution profiles of the saposin $\mathrm{B}^{\mathrm{W}}$-detergent co-assemblies are shown red, and dimeric saposin $\mathrm{B}^{\mathrm{W}}$ is shown in blue for comparison. The elution buffer used was $25 \mathrm{mM}$ Tris- $\mathrm{HCl}, \mathrm{pH} 7.5$, $150 \mathrm{mM} \mathrm{NaCl}$. 
A.
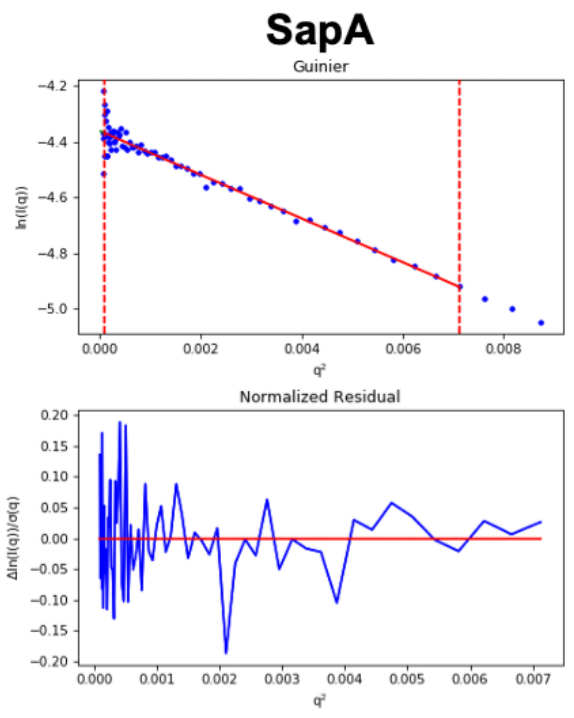

C.

SapA+MNA C13
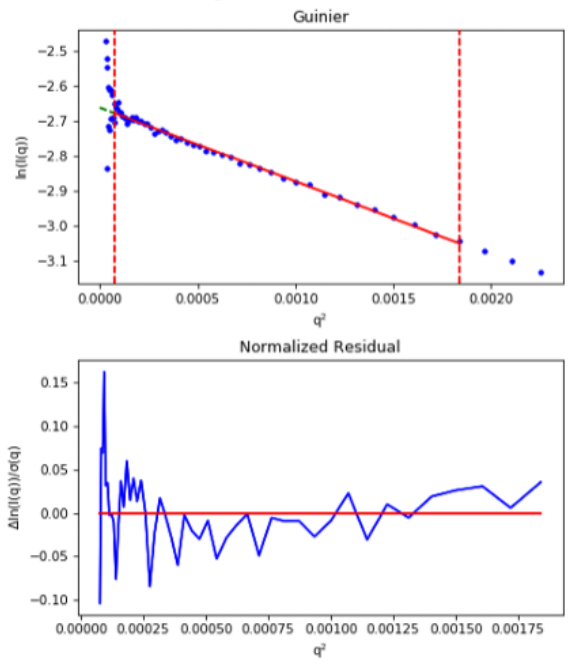

E.
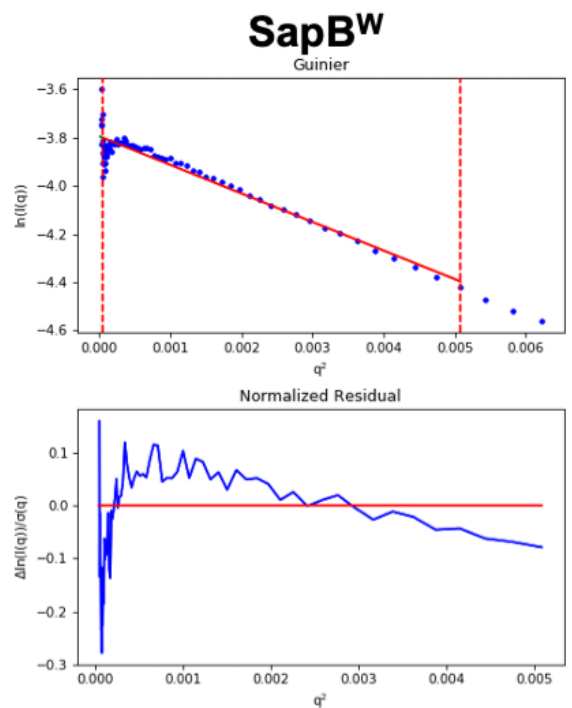

B.

SapA+MNA C12
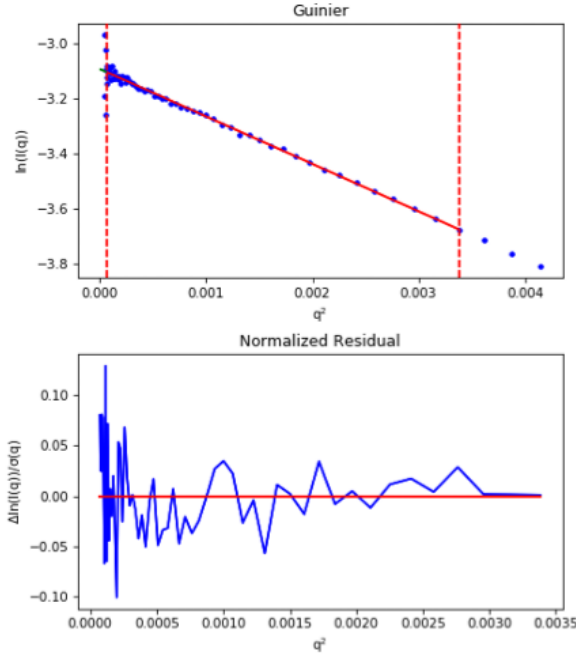

D.

SapA+LMNG
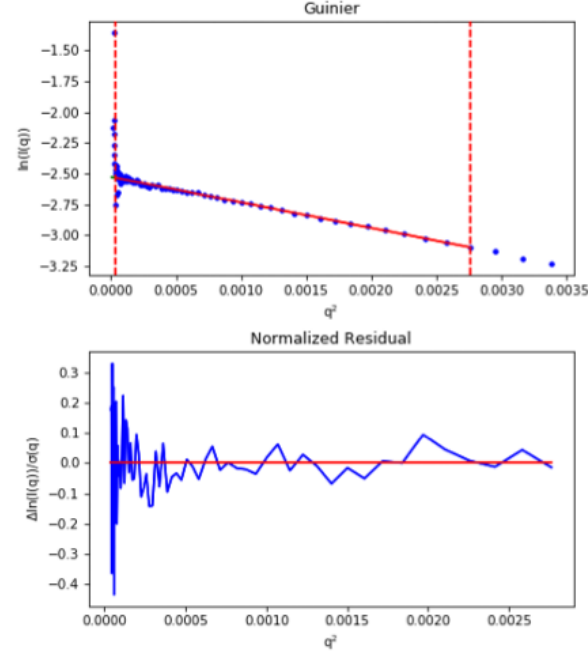

F.

SapBW+MNA C12
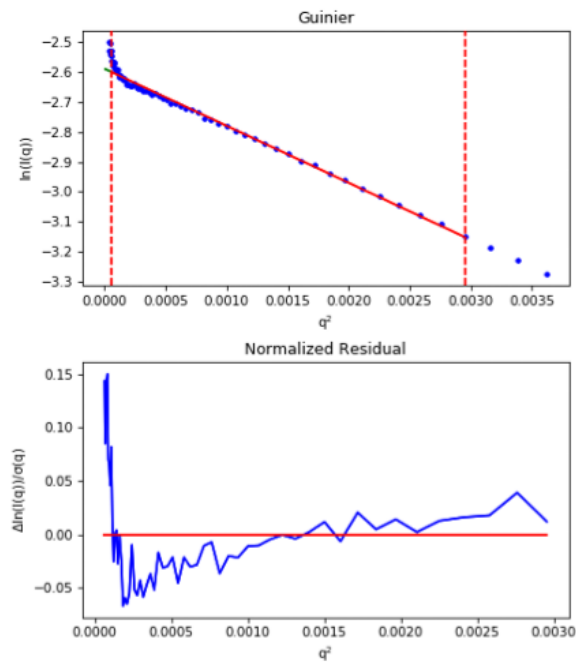
G.
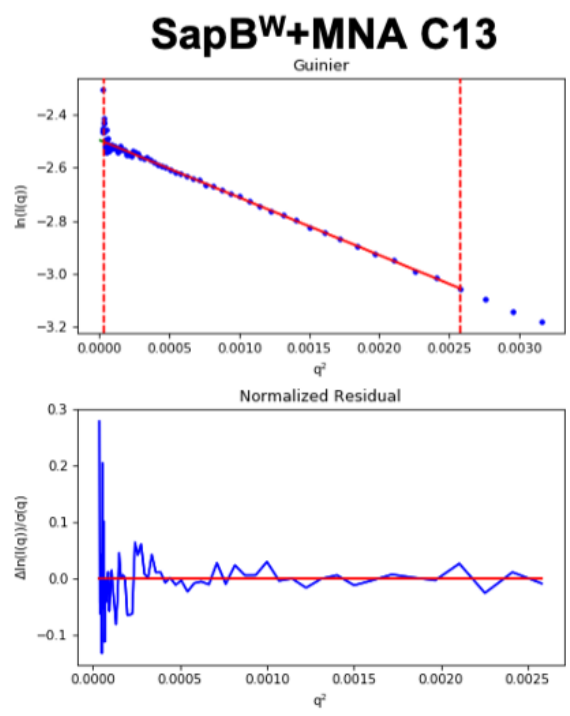

H.

SapB ${ }^{W}+$ LMNG

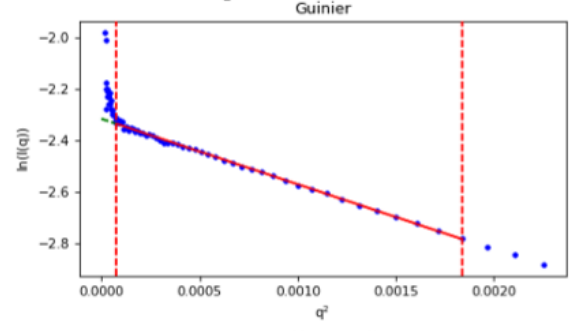

Normalized Residual

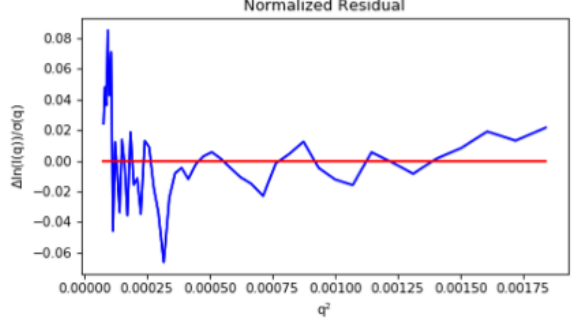

Figure S16. Guinier plots exported from RAW. ${ }^{6}$ Radius of gyration $\left(R_{g}\right)$ values were derived using the Guinier approximation that states that at low q-values, $I(q)=I o \exp \left(-R_{g}^{2} q^{2} / 3\right)$, where $\mathrm{I}_{\mathrm{o}}$ is the forward scattering, an indicator of molecular weight. $\mathrm{I}_{0}$ and $\mathrm{R}_{\mathrm{g}}$ values are determined by performing a linear fit to the plot of $\ln (I)$ vs. $q^{2}$. The Guinier fits for saposin $A(A)$, saposin $A+M N A$ C12 (B), saposin A+MNA C13 (C), saposin A+LMNG (D), saposin $B^{W}(E)$, saposin $B^{W}+M N A C 12$ $(\mathrm{F})$, saposin $\mathrm{B}^{\mathrm{W}}+\mathrm{MNA} \mathrm{C} 13(\mathrm{G})$, and saposin $\mathrm{B}^{\mathrm{W}}+\mathrm{LMNG}(\mathrm{H})$ are shown in the top plots and the bottom plots show the residuals of the fits. 


\section{References}

1. Ahn, V. E. Faull, K. F.; Whitlegge, J. P.; Fluharty, A. L. Privé, G. G. Crystal structure of saposin B reveals a dimeric shell for lipid binding. Proc. Natl. Acad. U.S.A. 2003, 100, 3843.

2. Rossmann, M.; Schultz-Helenbrok, R.; Behlke, J.; Remmel, N.; Alings, C.; Sandhoff, K.; Saenger, W.; Maier, T. Crystal structures of human saposins C and D: implications for lipid recognition and membrane interactions. Structure 2008, 16, 809-817.

3. Gerbai, A.; Gorelik, A.; Nager, B. Crystal structure of saposin D in an open conformation. J. Struc. Biol. 2018, 204, 145-150.

4. Popovic, K.; Holyoake, J.; Pomès, R.; Privé, G. G. Structure of saposin A lipoprotein discs. Proc. Natl. Acad. Sci. U. S. A. 2012, 109(8), 2908-2912.

5. Ahn, V. E.; Leyko, P.; Alattia, J. R.; Chen, L.; Privé, G. G. Crystal structures of saposins A and C.Protein Sci. 2006, 15, 1849-1857.

6. Svergun, D. I. Restoring low resolution structure of biological macromolecules from solution scattering using simulated annealing. Biophys J. 1999, 2879-2886.

7. Hopkins, J. B.; Gillilan, R. E.; Skou, S. BioXTAS RAW: improvements to an open-source program for small-angle X-ray scattering data reduction and analysis. J. Appl. Crystallogr. 2017, 50, 1545-1553.

8. Trewhella, J.; Duff, A. P.; Durand, D.I Gabel, F.; Guss, J. M.; Hendrickson, W. A.; Hura, G. L.; Jacques, D. A.; Kirby, N. M.; Kwan, A. H.; Pérez, J.; Pollack, L.; Ryam, T. M.; Sali, A.; Schneidman-Duhovny, D.; Schwede, T.; Svergun, D. I.; Sugiyama, M.; Tainer, J. A.; Vachette, P.; Westbrook, J.; Wihtten, A. E. 2017 publication guidelines for structural modelling of small-angle scattering data from biomolecules in solution: an update. Acta Cryst. 2017, D73, 710-728.

9. Pace, C. N.; Vajdos, F.; Fee, L.; Grimsley, G.; Gray, T. How to measure and predict the molar absorption coefficient of a protein. Protein Sci. 1995, 4, 2411-2423. 
10. Konarev, P.V.; Volkov, V. V.; Sokolova, A. V.; Koch, M. H. J.; Svergun, D. I. PRIMUS - a Windows-PC based system for small-angle scattering data analysis. J Appl. Cryst. 2003, $36,1277-1282$.

11. Svergun, D. I.; Barberato, C.; Koch, M. H. J. CRYSOL - a Program to Evaluate X-ray Solution Scattering of Biological Macromolecules from Atomic Coordinates. J. Appl. Cryst. $1995,28,768-773$. 